\title{
Per-Antenna Constant Envelope Precoding for Large Multi-User MIMO Systems
}

\author{
Saif Khan Mohammed and Erik G. Larsson
}

\section{Linköping University Post Print}

\section{Tweet}

N.B.: When citing this work, cite the original article.

(C2013 IEEE. Personal use of this material is permitted. However, permission to reprint/republish this material for advertising or promotional purposes or for creating new collective works for resale or redistribution to servers or lists, or to reuse any copyrighted component of this work in other works must be obtained from the IEEE.

Saif Khan Mohammed and Erik G. Larsson, Per-Antenna Constant Envelope Precoding for Large Multi-User MIMO Systems, 2013, IEEE Transactions on Communications, (61), 3, 1059-1071.

http://dx.doi.org/10.1109/TCOMM.2013.012913.110827

Postprint available at: Linköping University Electronic Press

http://urn.kb.se/resolve?urn=urn:nbn:se:liu:diva-93866 


\title{
Per-antenna Constant Envelope Precoding for Large Multi-User MIMO Systems
}

\author{
Saif Khan Mohammed and Erik G. Larsson
}

\begin{abstract}
We consider the multi-user MIMO broadcast channel with $M$ single-antenna users and $N$ transmit antennas under the constraint that each antenna emits signals having constant envelope (CE). The motivation for this is that CE signals facilitate the use of power-efficient RF power amplifiers. Analytical and numerical results show that, under certain mild conditions on the channel gains, for a fixed $M$, an array gain is achievable even under the stringent per-antenna $\mathrm{CE}$ constraint. Essentially, for a fixed $M$, at sufficiently large $N$ the total transmitted power can be reduced with increasing $N$ while maintaining a fixed information rate to each user. Simulations for the i.i.d. Rayleigh fading channel show that the total transmit power can be reduced linearly with increasing $N$ (i.e., an $O(N)$ array gain). We also propose a precoding scheme which finds near-optimal CE signals to be transmitted, and has $O(M N)$ complexity. Also, in terms of the total transmit power required to achieve a fixed desired information sum-rate, despite the stringent per-antenna CE constraint, the proposed CE precoding scheme performs close to the sum-capacity achieving scheme for an average-only total transmit power constrained channel.
\end{abstract}

Index Terms-Multi-user, constant envelope, per-antenna, large MIMO, GBC.

\section{INTRODUCTION}

We consider a Gaussian Broadcast Channel (GBC), wherein a base station (BS) having $N$ antennas communicates with $M$ single-antenna users in the downlink. Large antenna arrays at the BS has been of recent interest, due to their remarkable ability to suppress multi-user interference (MUI) with very simple precoding techniques [1]. Specifically, under an average only total transmit power constraint (APC), for a fixed $M$, a simple matched-filter precoder has been shown to achieve total MUI suppression in the limit as $N \rightarrow \infty$ [2]. Additionally, due to the inherent array power gain property ${ }^{1}$, large antenna arrays are also being considered as an enabler for reducing power consumption in wireless communications, especially since the operational power consumption at BS is becoming a matter of world-wide concern [4], [5].

Despite the benefits of large antenna arrays at the BS, practically building them would require cheap and powerefficient RF power amplifiers (PA's). In conventional BS,

Manuscript received Dec. 5, 2011; revised June 8, 2012 and Sept. 17, 2012; accepted Oct. 23, 2012. The editor coordinating the review of this paper and approving it for publication was Ali Ghrayeb.

The authors are with the Communication Systems Division, Dept. of Electrical Engineering (ISY), Linköping University, Linköping, Sweden. This work was supported by the Swedish Foundation for Strategic Research (SSF), ELLIIT. The work of Saif Khan Mohammed was partly supported by the Center for Industrial Information Technology at ISY, Linköping University (CENIIT). Parts of the results in this paper were presented at IEEE ICASSP 2012 [15]. Also, the simpler special case of $M=1$ (i.e., single-user) has been studied by us in much greater detail in [16].

${ }^{1}$ Under an APC constraint, for a fixed $M$ and a fixed desired information sum-rate, the required total transmit power decreases with increasing $N$ [3]. power-inefficient PA's account for about 40-50 percent of the total operational power consumption [5]. With current technology, power-efficient RF components are generally nonlinear. The type of transmitted signal that facilitates the use of most power-efficient/non-linear RF components, is a constant envelope (CE) signal. In this paper, we therefore consider a GBC, where the amplitude of the signal transmitted from each BS antenna is constant and independent of the channel realization. We only consider the discrete-time complex baseband equivalent channel model, where we aim to restrict the discrete-time per-antenna channel input to have no amplitude variations. Compared to precoding methods which result in large amplitude-variations in the discrete-time channel input, the CE precoding method proposed in this paper is expected to result in continuous-time transmit signals which have a significantly improved peak-to-average-power-ratio (PAPR). However, this does not necessarily mean that the proposed precoding method will result in continuous-time transmit signals having a perfectly constant envelope. Generation of perfectly constant envelope continuous-time transmit signals constitutes future work for us. One possible method to generate almost constant-envelope continuous-time signals could be to constrain the phase variation between consecutive constant amplitude baseband symbols of the discrete-time channel input.

Since the per-antenna CE constraint is much more restrictive than APC, in this paper we investigate as to whether MUI suppression and array power gain can still be achieved under the stringent per-antenna CE constraint. To the best of our knowledge, there is no reported work which addresses this question. Most reported work on per-antenna communication consider an average-only or a peak-only power constraint (see [6], [7] and references therein). In this paper, firstly, we derive expressions for the MUI at each user under the per-antenna CE constraint, and then propose a low-complexity CE precoding scheme with the objective of minimizing the MUI energy at each user. For a given vector of information symbols to be communicated to the users, the proposed precoding scheme chooses per-antenna CE transmit signals in such a way that the MUI energy at each user is small (i.e., of the same order or less than the variance of the additive white Gaussian noise). Throughout the paper, we assume that such large antenna systems will not operate in a regime where the MUI energy is significantly larger than the AWGN variance, since it is highly power-inefficient to do so [8].

Secondly, under certain mild channel conditions (including i.i.d. fading), using a novel probabilistic approach, we analytically show that, MUI suppression can be achieved even under the stringent per-antenna CE constraint. Specifically, 
for a fixed $M$ and fixed user information symbol alphabets, an arbitrarily low MUI energy can be guaranteed at each user, by choosing a sufficiently large $N$. Our analysis further reveals that, with a fixed $M$ and increasing $N$, the total transmitted power can be reduced while maintaining a constant signal-tointerference-and-noise-ratio (SINR) level at each user.

Thirdly, through simulation, we confirm our analytical observations for the i.i.d. Rayleigh fading channel. For the proposed CE precoder, we numerically compute an achievable ergodic information sum-rate, and observe that, for a fixed $M$ and a fixed desired ergodic sum-rate, the required total transmit power reduces linearly with increasing $N$ (i.e., an $O(N)$ array power gain is achieved under the per-antenna CE constraint). We also observe that, to achieve a given desired ergodic information sum-rate, compared to the optimal GBC sum-capacity achieving scheme under APC, the extra total transmit power required by the proposed $\mathrm{CE}$ precoding scheme is small (roughly $2.0 \mathrm{~dB}$ for sufficiently large $N$ ).

Notation: $\mathbb{C}$ and $\mathbb{R}$ denote the set of complex and real numbers. $|x|, x^{*}$ and $\arg (x)$ denote the absolute value, complex conjugate and argument of $x \in \mathbb{C}$ respectively. $\|\mathbf{h}\|^{2} \triangleq \sum_{i}\left|h_{i}\right|^{2}$ denotes the squared Euclidean-norm of $\mathbf{h}=\left(h_{1}, \cdots, h_{N}\right) \in \mathbb{C}^{N} \cdot \mathbb{E}[\cdot]$ denotes the expectation operator. Abbreviations: r.v. (random variable), bpcu (bits-perchannel-use), p.d.f. (probability density function).

\section{SySTEM MODEL}

Let the complex channel gain between the $i$-th BS antenna and the $k$-th user be denoted by $h_{k, i}$. The vector of channel gains from the $\mathrm{BS}$ antennas to the $k$-th user is denoted by $\mathbf{h}_{k}=\left(h_{k, 1}, h_{k, 2}, \cdots, h_{k, N}\right)^{T} . \mathbf{H} \in \mathbb{C}^{M \times N}$ is the channel gain matrix with $h_{k, i}$ as its $(k, i)$-th entry. Let $x_{i}$ denote the complex symbol transmitted from the $i$-th BS antenna. Further, let $P_{T}$ denote the average total power transmitted from all the BS antennas. Under APC, we must have $\mathbb{E}\left[\sum_{i=1}^{N}\left|x_{i}\right|^{2}\right]=$ $P_{T}$, whereas under the per-antenna CE constraint we have $\left|x_{i}\right|^{2}=P_{T} / N, i=1,2, \cdots, N$ which is clearly a more stringent constraint compared to APC. Further, due to the per-antenna CE constraint, it is clear that $x_{i}$ is of the form $x_{i}=\sqrt{P_{T} / N} e^{j \theta_{i}}$, where $\theta_{i}$ is the phase of $x_{i}$. Under CE transmission, the symbol received by the $k$-th user is therefore given by

$$
y_{k}=\sqrt{\frac{P_{T}}{N}} \sum_{i=1}^{N} h_{k, i} e^{j \theta_{i}}+w_{k}, k=1,2, \ldots, M
$$

where $w_{k} \sim \mathcal{C N}\left(0, \sigma^{2}\right)$ is the AWGN noise at the $k$-th receiver. For the sake of notation, let $\Theta=\left(\theta_{1}, \cdots, \theta_{N}\right)^{T}$ denote the vector of transmitted phase angles. Let $\mathbf{u}=$ $\left(\sqrt{E_{1}} u_{1}, \cdots, \sqrt{E_{M}} u_{M}\right)^{T}$ be the vector of scaled information symbols, with $u_{k} \in \mathcal{U}_{k}$ denoting the information symbol to be communicated to the $k$-th user. Here $\mathcal{U}_{k}$ denotes the unit average energy information alphabet of the $k$-th user. $E_{k}, k=1,2, \ldots, M$ denotes the information symbol energy for each user. Also, let $\mathcal{U} \triangleq \sqrt{E_{1}} \mathcal{U}_{1} \times \sqrt{E_{2}} \mathcal{U}_{2} \times \cdots \times \sqrt{E_{M}} \mathcal{U}_{M}$. Subsequently, in this paper, we are interested in scenarios where $M$ is fixed and $N$ is allowed to increase. Also, throughout this paper, for a fixed $M$, the alphabets $\mathcal{U}_{1}, \cdots, \mathcal{U}_{M}$ are also fixed and do not change with increasing $N$.

We stress that $\mathrm{CE}$ transmission is entirely different from equal gain transmission (EGT). We explain this difference for the simple single-user scenario $(M=1)$. In EGT a unit average energy complex information symbol $u$ is communicated to the user by transmitting $x_{i}=w_{i} u$ from the $i$-th transmit antenna (with $\left|w_{1}\right|=\cdots=\left|w_{N}\right|=\sqrt{P_{T} / N}$ ), and therefore the amplitude of the signal transmitted from each antenna is not constant but varies with the amplitude of $u$ $\left(\left|x_{i}\right|=\sqrt{P_{T} / N}|u|\right)$. In contrast, the CE precoding method proposed in this paper (Section III-B) transmits a constant amplitude signal from each antenna (i.e., $\sqrt{P_{T} / N} e^{j \theta_{i}}$ from the $i$-th antenna), where the transmit phase angles $\theta_{1}, \cdots, \theta_{N}$ are chosen in such a way that the noise-free received signal is a known constant times the desired information symbol $u$.

\section{MUi AnAlysis AND the Proposed CE Precoder}

For any given information symbol vector $\mathbf{u}$ to be communicated, with $\Theta$ as the transmitted phase angle vector, using (1) the received signal at the $k$-th user can be expressed as

$$
\begin{aligned}
& y_{k}=\sqrt{P_{T}} \sqrt{E_{k}} u_{k}+\sqrt{P_{T}} s_{k}+w_{k}, \\
& s_{k} \triangleq\left(\frac{\sum_{i=1}^{N} h_{k, i} e^{j \theta_{i}}}{\sqrt{N}}-\sqrt{E_{k}} u_{k}\right)
\end{aligned}
$$

where $\sqrt{P_{T}} s_{k}$ is the MUI term at the $k$-th user. In this section we aim to get a better understanding of the MUI energy level at each user, for any general CE precoding scheme where the signal transmitted from each BS antenna has constant envelope. Towards this end, we firstly study the range of values taken by the noise-free received signal at the users (scaled down by $\sqrt{P_{T}}$ ). This range of values is given by the set

$$
\begin{gathered}
\mathcal{M}(\mathbf{H}) \triangleq\left\{\mathbf{v}=\left(v_{1}, \cdots, v_{M}\right) \in \mathbb{C}^{M} \mid v_{k}=\frac{\sum_{i=1}^{N} h_{k, i} e^{j \theta_{i}}}{\sqrt{N}}\right. \\
\left.\theta_{i} \in[-\pi, \pi), i=1, \ldots, N\right\}
\end{gathered}
$$

For any vector $\mathbf{v}=\left(v_{1}, v_{2}, \cdots, v_{M}\right)^{T} \in \mathcal{M}(\mathbf{H})$, from (3) it follows that there exists a $\Theta^{v}=\left(\theta_{1}^{v}, \cdots, \theta_{N}^{v}\right)^{T}$ such that $v_{k}=$ $\frac{\sum_{i=1}^{N} h_{k, i} e^{j \theta_{i}^{v}}}{\sqrt{N}}, k=1,2, \ldots, M$. This sum can be expressed as a sum of $N / M$ terms (without loss of generality let us assume that $N / M$ is integral only for the argument presented here)

$$
\begin{aligned}
& v_{k}=\sum_{q=1}^{N / M} v_{k}^{q}, \\
& v_{k}^{q} \triangleq\left(\sum_{r=(q-1) M+1}^{q M} h_{k, r} e^{j \theta_{r}^{v}}\right) / \sqrt{N}, q=1, \ldots, \frac{N}{M} .
\end{aligned}
$$


From (4) it follows that $\mathcal{M}(\mathbf{H})$ can be expressed as a directsum of $N / M$ sets, i.e.

$$
\begin{gathered}
\mathcal{M}(\mathbf{H})=\mathcal{M}\left(\mathbf{H}^{(1)}\right) \oplus \mathcal{M}\left(\mathbf{H}^{(2)}\right) \oplus \cdots \oplus \mathcal{M}\left(\mathbf{H}^{(N / M)}\right) \\
\mathcal{M}\left(\mathbf{H}^{(q)}\right) \triangleq\left\{\mathbf{v}=\left(v_{1}, \cdots, v_{M}\right) \in \mathbb{C}^{M} \mid\right. \\
\left.v_{k}=\frac{\sum_{i=1}^{M} h_{k,(q-1) M+i} e^{j \theta_{i}}}{\sqrt{N}}, \theta_{i} \in[-\pi, \pi)\right\} \\
, q=1, \ldots, N / M
\end{gathered}
$$

where $\mathbf{H}^{(q)}$ is the sub-matrix of $\mathbf{H}$ containing only the columns numbered $(q-1) M+1,(q-1) M+2, \cdots, q M$. $\mathcal{M}\left(\mathbf{H}^{(q)}\right) \subset \mathbb{C}^{M}$ is the dynamic range of the received noise-free signals when only the $M$ BS antennas numbered $(q-1) M+1,(q-1) M+2, \cdots, q M$ are used and the remaining $N-M$ antennas are inactive. If the statistical distribution of the channel gain vector from a BS antenna to all the users is identical for all the BS antennas (as in i.i.d. channels), then, on an average the sets $\mathcal{M}\left(\mathbf{H}^{(q)}\right), q=1, \ldots, N / M$ would all have similar topological properties. Since, $\mathcal{M}(\mathbf{H})$ is a directsum of $N / M$ topologically similar sets, it is expected that for a fixed $M$, on an average the region $\mathcal{M}(\mathbf{H})$ expands with increasing $N$. Specifically, for a fixed $M$ and increasing $N$, the maximum Euclidean length of any vector in $\mathcal{M}(\mathbf{H})$ grows as $O(\sqrt{N})$, since $\mathcal{M}(\mathbf{H})$ is a direct-sum of $O(N)$ topologically similar sets $\left(\mathcal{M}\left(\mathbf{H}^{(q)}\right), q=1,2, \ldots, N / M\right)$ with the maximum Euclidean length of any vector in $\mathcal{M}\left(\mathbf{H}^{(q)}\right)$ being $O(1 / \sqrt{N})$ (note that in the definition of $\mathcal{M}\left(\mathbf{H}^{(q)}\right)$ in (5), each component of any vector $\mathbf{v} \in \mathcal{M}\left(\mathbf{H}^{(q)}\right)$ is scaled down by $\sqrt{N})$. Also, for a fixed $M$ and increasing $N$, since $\mathcal{M}(\mathbf{H})$ is a direct-sum of $N / M$ similar sets, it is expected that the set $\mathcal{M}(\mathbf{H})$ becomes increasingly dense (i.e., the number of elements of $\mathcal{M}(\mathbf{H})$ in a fixed volume in $\mathbb{C}^{M}$ is expected to increase with increasing $N$ ). The above discussion leads us to the following results in Section III-A and III-C.

\section{A. Diminishing MUI with increasing $N$, for fixed $M$ and fixed $E_{k}(k=1, \ldots, M)$}

For a fixed $M$ and fixed $E_{k}$, the information alphabets and the information symbol energies are fixed. However, since increasing $N$ (with fixed $M$ ) is expected to enlarge the set $\mathcal{M}(\mathbf{H})$ and make it increasingly denser, it is highly probable that at sufficiently large $N$, for any fixed information symbol vector $\mathbf{u}=\left(\sqrt{E_{1}} u_{1}, \cdots, \sqrt{E_{M}} u_{M}\right)^{T} \in \mathcal{U}$ there exists a vector $\mathbf{v} \in \mathcal{M}(\mathbf{H})$ such that $\mathbf{v}$ is very close to $\mathbf{u}$ in terms of Euclidean distance. This then implies that, with increasing $N$ and fixed $M$, for any $\mathbf{u} \in \mathcal{U}$ there exists a transmit phase angle vector $\Theta$ such that the sum of the MUI energy for all users is small compared to the AWGN variance at the receiver. Hence, for a fixed $M$ and fixed $E_{k}$, it is expected that the MUI energy for each user decreases with increasing $N$.

This is in fact true, as we prove it formally for channels satisfying the following mild conditions. Specifically for a fixed $M$, we consider a sequence of channel gain matrices
$\left\{\mathbf{H}_{N}\right\}_{N=M}^{\infty}$ satisfying

$$
\begin{aligned}
\lim _{N \rightarrow \infty} \frac{\left|\mathbf{h}_{k}^{(N)} \mathbf{h}_{l}^{H}\right|}{N} & =0, k \neq l \text { (As.1) } \\
\lim _{N \rightarrow \infty} \frac{\sum_{i=1}^{N}\left|h_{k_{1}, i}^{(N)}\right|\left|h_{l_{1}, i}^{(N)}\right|\left|h_{k_{2}, i}^{(N)}\right|\left|h_{l_{2}, i}^{(N)}\right|}{N^{2}} & =0, \text { (As.2) } \\
\lim _{N \rightarrow \infty} \frac{\left\|\mathbf{h}_{k}^{(N)}\right\|^{2}}{N} & =c_{k}, \text { (As.3) } \\
k, l, k_{1}, l_{1}, k_{2}, l_{2} \in(1,2, \ldots, M) & \text { (6) }
\end{aligned}
$$

where $c_{k}$ are positive constants, $\mathbf{h}_{k}^{(N)}$ denotes the $k$-th row of $\mathbf{H}_{N}$ and $h_{k, i}^{(N)}$ denotes the $i$-th component of $\mathbf{h}_{k}^{(N)}$. From the law of large numbers, it follows that i.i.d. channels satisfy these conditions with probability one [13]. Physical measurements of the channel characteristics with large antenna arrays at the BS have revealed closeness to the i.i.d. fading model, as long as the BS antennas are sufficiently spaced apart (usually half of the carrier wavelength) [14], [1].

Theorem 1: For a fixed $M$ and increasing $N$, consider a sequence of channel gain matrices $\left\{\mathbf{H}_{N}\right\}_{N=M}^{\infty}$ satisfying the mild conditions in (6). For any given fixed finite alphabet $\mathcal{U}$ (fixed $E_{k}, k=1, \ldots, M$ ) and any given $\Delta>0$, there exist a corresponding integer $N\left(\left\{\mathbf{H}_{N}\right\}, \mathcal{U}, \Delta\right)$ such that with $N \geq N\left(\left\{\mathbf{H}_{N}\right\}, \mathcal{U}, \Delta\right)$ and $\mathbf{H}_{N}$ as the channel gain matrix, for any $\mathbf{u} \in \mathcal{U}$ to be communicated, there exist a phase angle vector $\Theta_{N}^{u}(\Delta)=\left(\theta_{1}^{u}(\Delta), \cdots, \theta_{N}^{u}(\Delta)\right)^{T}$ which when transmitted, results in the MUI energy at each user being upper bounded by $2 \Delta^{2}$, i.e.

$$
\left|\frac{\sum_{i=1}^{N} h_{k, i}^{(N)} e^{j \theta_{i}^{u}(\Delta)}}{\sqrt{N}}-\sqrt{E_{k}} u_{k}\right|^{2} \leq 2 \Delta^{2}, k=1, \ldots, M .
$$

Proof - The proof relies on technical results in Theorem 3 (stated and proved in Appendix A) and Theorem 2 (stated and proved below). All these results assume a fixed $M$ (number of user terminals) and increasing $N$ (number of BS antennas). These results are stated for a fixed sequence of channel matrices $\left\{\mathbf{H}_{N}\right\}_{N=M}^{\infty}$, fixed information alphabets $\mathcal{U}_{1}, \cdots, \mathcal{U}_{M}$ and fixed information symbol energy $E_{1}, \cdots, E_{M}$. Further, the sequence of channel matrices $\left\{\mathbf{H}_{N}\right\}_{N=M}^{\infty}$ is assumed to satisfy the conditions in (6) and the information alphabets are assumed to be finite/discrete. The proofs use a novel probabilistic approach, treating the transmitted phase angles as random variables. We now present the proof of Theorem 1 .

Let us consider a probability space with the transmitted phase angles $\theta_{i}, i=1,2, \ldots, N$ being i.i.d. r.v's uniformly distributed in $[-\pi, \pi)$. For a given sequence of channel matrices $\left\{\mathbf{H}_{N}\right\}$, we define a corresponding sequence of r.v's $\left\{\mathbf{z}_{N}\right\}$, with $\mathbf{z}_{N} \triangleq\left(z_{1}^{I^{(N)}}, z_{1}^{Q^{(N)}}, \ldots, z_{M}^{I^{(N)}}, z_{M}^{Q^{(N)}}\right) \in \mathbb{R}^{2 M}$, where we have

$$
\begin{aligned}
& z_{k}^{I^{(N)}} \triangleq \operatorname{Re}\left(\frac{\sum_{i=1}^{N} h_{k, i}^{(N)} e^{j \theta_{i}}}{\sqrt{N}}\right), z_{k}^{Q^{(N)}} \triangleq \operatorname{Im}\left(\frac{\sum_{i=1}^{N} h_{k, i}^{(N)} e^{j \theta_{i}}}{\sqrt{N}}\right) \\
&, k=1, \ldots, M .
\end{aligned}
$$

From Theorem 3 it follows that, for any channel sequence $\left\{\mathbf{H}_{N}\right\}$ satisfying the conditions in (6), as $N \rightarrow \infty$ (with fixed $M$ ), the corresponding sequence of r.v's $\left\{\mathbf{z}_{N}\right\}$ converges in distribution to a $2 M$-dimensional real Gaussian random 


$$
\mathcal{B}_{\Delta}(\mathbf{u}) \triangleq\left\{\mathbf{b}=\left(b_{1}^{I}, b_{1}^{Q}, \cdots, b_{M}^{I}, b_{M}^{Q}\right)^{T} \in \mathbb{R}^{2 M}|| b_{k}^{I}-\sqrt{E_{k}} u_{k}^{I}|\leq \Delta,| b_{k}^{Q}-\sqrt{E_{k}} u_{k}^{Q} \mid \leq \Delta, k=1,2, \ldots, M\right\}
$$

vector $X=\left(X_{1}^{I}, X_{1}^{Q}, \cdots, X_{M}^{I}, X_{M}^{Q}\right)^{T}$ with independent zero-mean components and $\operatorname{var}\left(X_{k}^{I}\right)=\operatorname{var}\left(X_{k}^{Q}\right)=c_{k} / 2, k=$ $1,2, \ldots, M$. For a given $\mathbf{u}=\left(\sqrt{E_{1}} u_{1}, \cdots, \sqrt{E_{M}} u_{M}\right)^{T} \in \mathcal{U}$, and $\Delta>0$, we next consider the box $\mathcal{B}_{\Delta}(\mathbf{u})$ defined in (9) (at the top of this page), where $u_{k}^{I} \triangleq \operatorname{Re}\left(u_{k}\right), u_{k}^{Q} \triangleq \operatorname{Im}\left(u_{k}\right)$. The box $\mathcal{B}_{\Delta}(\mathbf{u})$ contains all those vectors in $\mathbb{R}^{2 M}$ whose component-wise displacement from $\mathbf{u}$ is upper bounded by $\Delta$. Using the fact that $\mathbf{z}_{N}$ converges in distribution to a Gaussian r.v. with $\mathbb{R}^{2 M}$ as its range space, in Theorem 2 it is shown that, for any $\Delta>0$, there exist an integer $N\left(\left\{\mathbf{H}_{N}\right\}, \mathcal{U}, \Delta\right)$, such that for all $N \geq N\left(\left\{\mathbf{H}_{N}\right\}, \mathcal{U}, \Delta\right)$

$$
\operatorname{Prob}\left(\mathbf{z}_{N} \in \mathcal{B}_{\Delta}(\mathbf{u})\right)>0, \forall \mathbf{u} \in \mathcal{U} \text {. }
$$

Since the probability that $\mathbf{z}_{N}$ lies in the box $\mathcal{B}_{\Delta}(\mathbf{u})$ is strictly positive for all $\mathbf{u} \in \mathcal{U}$, from the definitions of $\mathcal{B}_{\Delta}(\mathbf{u})$ in (9) and $\mathbf{z}_{N}$ in (8) it follows that, for any $\mathbf{u} \in \mathcal{U}$ there exist a phase angle vector $\Theta_{N}^{u}(\Delta)=\left(\theta_{1}^{u}(\Delta), \cdots, \theta_{N}^{u}(\Delta)\right)^{T}$ such that

$$
\begin{gathered}
\left|\operatorname{Re}\left(\frac{\sum_{i=1}^{N} h_{k, i}^{(N)} e^{j \theta_{i}^{u}(\Delta)}}{\sqrt{N}}\right)-\sqrt{E_{k}} u_{k}^{I}\right| \leq \Delta, \\
\left|\operatorname{Im}\left(\frac{\sum_{i=1}^{N} h_{k, i}^{(N)} e^{j \theta_{i}^{u}(\Delta)}}{\sqrt{N}}\right)-\sqrt{E_{k}} u_{k}^{Q}\right| \leq \Delta
\end{gathered}
$$

for all $k=1,2, \cdots, M$, which then implies (7).

Since Theorem 1 is valid for any $\Delta>0$ and (7) holds for all $N \geq N\left(\left\{\mathbf{H}_{N}\right\}, \mathcal{U}, \Delta\right)$, we can satisfy (7) for any arbitrarily small $\Delta>0$ by having $N \geq N\left(\left\{\mathbf{H}_{N}\right\}, \mathcal{U}, \Delta\right)$ i.e., a sufficiently large $N$. Hence, the MUI energy at each user can be guaranteed to be arbitrarily small by having a sufficiently large $N$. Theorem 1 therefore motivates us to propose precoding techniques which can achieve small MUI energy levels.

An essential part of the proof for Theorem 1 was the positivity of the box event probability $\operatorname{Prob}\left(\mathbf{z}_{N} \in \mathcal{B}_{\Delta}(\mathbf{u})\right)$, when $N$ is sufficiently large. In the following theorem, we formally state and prove the positivity of the box event probability.

Theorem 2: For a given channel sequence $\left\{\mathbf{H}_{N}\right\}_{N=M}^{\infty}$ satisfying (6) and a given fixed finite alphabet set $\mathcal{U}$, for any $\Delta>0$, there exist a corresponding integer $N\left(\left\{\mathbf{H}_{N}\right\}, \mathcal{U}, \Delta\right)$, such that for all $N \geq N\left(\left\{\mathbf{H}_{N}\right\}, \mathcal{U}, \Delta\right)$ (with fixed $M$ )

$$
\operatorname{Prob}\left(\mathbf{z}_{N} \in \mathcal{B}_{\Delta}(\mathbf{u})\right)>0, \forall \mathbf{u} \in \mathcal{U} \text {. }
$$

where $\mathcal{B}_{\Delta}(\mathbf{u})$ is defined in (9).

Proof - We consider the probability that a $n$-dimensional real r.v. $\mathbf{X}=\left(X_{1}, X_{2}, \cdots, X_{n}\right)$ lies in a $n$-dimensional box centered at $\boldsymbol{\alpha}=\left(\alpha_{1}, \ldots, \alpha_{n}\right) \in \mathbb{R}^{n}$ and denoted by $C(\Delta, \boldsymbol{\alpha})=\left\{\left(x_{1}, x_{2}, \cdots, x_{n}\right) \in \mathbb{R}^{n} \mid \alpha_{k}-\Delta \leq x_{k} \leq\right.$ $\left.\alpha_{k}+\Delta, k=1,2, \ldots, n\right\}$. For notational convenience, we refer to $\alpha_{k}+\Delta$ and $\alpha_{k}-\Delta$ as the corresponding "upper" and "lower" limits for the $k$-th coordinate. The probability that $\mathbf{X}$ lies in the box $C(\Delta, \boldsymbol{\alpha})$ is given by the expansion

$$
\operatorname{Prob}(\mathbf{X} \in C(\Delta, \boldsymbol{\alpha}))=\sum_{k=0}^{n}(-1)^{k} T_{k}(\Delta, \boldsymbol{\alpha})
$$

where $T_{k}(\Delta, \boldsymbol{\alpha})$ is the probability that the r.v. $\left(X_{1}, X_{2}, \cdots, X_{n}\right)$ belongs to a sub-region of $\left\{\left(x_{1}, \cdots, x_{n}\right) \in \mathbb{R}^{n} \mid x_{l} \leq \alpha_{l}+\Delta, l=1,2, \ldots, n\right\}$, where exactly $k$ coordinates are less than their corresponding "lower" limit and the remaining $n-k$ coordinates are less than their corresponding "upper" limit. Specifically, $T_{k}(\Delta, \boldsymbol{\alpha})$ is given $b y^{2}$

$$
\begin{aligned}
T_{k}(\Delta, \boldsymbol{\alpha})=\sum_{i_{1}=1}^{n} \sum_{i_{2}=i_{1}+1}^{n} \cdots \sum_{i_{k}=i_{k-1}+1}^{n} \operatorname{Prob}\left(X_{r} \leq \alpha_{r}-\Delta\right. \\
\quad \forall r \in\left\{i_{1}, i_{2}, \cdots, i_{k}\right\}, \\
\left.X_{r} \leq \alpha_{r}+\Delta \quad \forall r \notin\left\{i_{1}, i_{2}, \cdots, i_{k}\right\}\right)(14)
\end{aligned}
$$

Using the expansion in (13), the probability of the box event $\left\{\mathbf{z}_{N} \in \mathcal{B}_{\Delta}(\mathbf{u})\right\}$ can be expressed as

$$
\begin{aligned}
& \operatorname{Prob}\left(\mathbf{z}_{N} \in \mathcal{B}_{\Delta}(\mathbf{u})\right)= \\
& \operatorname{Prob}\left(\left(\sqrt{E_{k}} u_{k}^{I}-\Delta\right) \leq z_{k}^{I(N)} \leq\left(\sqrt{E_{k}} u_{k}^{I}+\Delta\right),\right. \\
& \left.\left(\sqrt{E_{k}} u_{k}^{Q}-\Delta\right) \leq z_{k}^{Q^{(N)}} \leq\left(\sqrt{E_{k}} u_{k}^{Q}+\Delta\right), k=1,2, \ldots, M\right) \\
& =\sum_{k=0}^{2 M}(-1)^{k} \sum_{i_{1}=1}^{2 M} \sum_{i_{2}=i_{1}+1}^{2 M} \cdots \sum_{i_{k}=i_{k-1}+1}^{2 M} \operatorname{Prob}\left(z_{l}^{(N)} \leq \sqrt{E_{l}} u_{l}-\Delta\right. \\
& \quad \forall l \in\left\{i_{1}, i_{2}, \cdots, i_{k}\right\}, \\
& \left.z_{l}^{(N)} \leq \sqrt{E_{l}} u_{l}+\Delta \quad \forall l \notin\left\{i_{1}, i_{2}, \cdots, i_{k}\right\}\right)(15)
\end{aligned}
$$

where $z_{l}^{(N)}$ is the $l$-th component of $\mathbf{z}_{N}$ (i.e., $z_{l}^{(N)}=z_{l / 2}^{Q^{(N)}}$ for even $l$, and $z_{l}^{(N)}=z_{(l+1) / 2}^{I^{(N)}}$ for odd $l$ ) and $u_{l}$ is the $l$-th component of the vector $\left(u_{1}^{I}, u_{1}^{Q}, u_{2}^{I}, u_{2}^{Q}, \cdots, u_{M}^{I}, u_{M}^{Q}\right)^{T}$. For notational convenience we define

$$
\begin{gathered}
T^{(N)}\left(k, i_{1}, i_{2}, \cdots, i_{k}, \mathbf{u}, \Delta\right) \triangleq \\
\operatorname{Prob}\left(z_{l}^{(N)} \leq \sqrt{E_{l}} u_{l}-\Delta \forall l \in\left\{i_{1}, i_{2}, \cdots, i_{k}\right\},\right. \\
\left.z_{l}^{(N)} \leq \sqrt{E_{l}} u_{l}+\Delta \forall l \notin\left\{i_{1}, i_{2}, \cdots, i_{k}\right\}\right) \\
1 \leq i_{1}<i_{2}<\cdots<i_{k} \leq 2 M, 0 \leq k \leq 2 M .
\end{gathered}
$$

Let $\mathbf{Y}=\left(Y_{1}, Y_{2}, \cdots, Y_{2 M}\right)$ denote a multivariate $2 M$ dimensional real Gaussian r.v. with independent zero mean components and $\operatorname{var}\left(Y_{2 k-1}\right)=\operatorname{var}\left(Y_{2 k}\right)=c_{k} / 2, k=$ $1,2, \ldots, M$. From Theorem 3 (Appendix A) it follows that the c.d.f. of $\mathbf{z}_{N}$ converges to the c.d.f. of $\mathbf{Y}$ as $N \rightarrow \infty$. This convergence in distribution implies that, for any given arbitrary $\delta>0$, for each term

\footnotetext{
2 As an example, for $n=2$, we have $\operatorname{Prob}\left(\alpha_{1}-\Delta \leq X_{1} \leq \alpha_{1}+\right.$ $\left.\Delta, \alpha_{2}-\Delta \leq X_{2} \leq \alpha_{2}+\Delta\right)=T_{0}(\Delta, \boldsymbol{\alpha})-T_{1}(\Delta, \boldsymbol{\alpha})+T_{2}(\Delta, \boldsymbol{\alpha})$, where $T_{0}(\Delta, \boldsymbol{\alpha}) \triangleq \operatorname{Prob}\left(X_{1} \leq \alpha_{1}+\Delta, X_{2} \leq \alpha_{2}+\Delta\right), T_{2}(\Delta, \boldsymbol{\alpha}) \triangleq$ $\operatorname{Prob}\left(X_{1} \leq \alpha_{1}-\Delta, X_{2} \leq \alpha_{2}-\Delta\right)$, and $T_{1}(\Delta, \boldsymbol{\alpha}) \triangleq \operatorname{Prob}\left(X_{1} \leq\right.$ $\left.\alpha_{1}+\Delta, \bar{X}_{2} \leq \alpha_{2}-\Delta\right)+\operatorname{Prob}\left(X_{1} \leq \alpha_{1}-\Delta, X_{2} \leq \alpha_{2}+\Delta\right)$.
} 


$$
\begin{array}{r}
\mid T^{(N)}\left(k, i_{1}, i_{2}, \cdots, i_{k}, \mathbf{u}, \Delta\right)-\operatorname{Prob}\left(Y_{l} \leq \sqrt{E_{l}} u_{l}-\Delta \forall l \in\left\{i_{1}, i_{2}, \cdots, i_{k}\right\},\right. \\
\left.Y_{l} \leq \sqrt{E_{l}} u_{l}+\Delta \forall l \notin\left\{i_{1}, i_{2}, \cdots, i_{k}\right\}\right) \mid \leq \delta . \\
g\left(\left\{\mathbf{H}_{N}\right\}, \mathbf{u}, \Delta, \delta\right) \triangleq \max _{k=0,1, \cdots, 2 M} \max _{1 \leq i_{1}<i_{2}<\cdots<i_{k} \leq 2 M} N\left(k, i_{1}, i_{2}, \cdots, i_{k}, \delta, \mathbf{u}, \Delta\right)
\end{array}
$$

$$
\begin{aligned}
& \left|\operatorname{Prob}\left(\mathbf{z}_{N} \in \mathcal{B}_{\Delta}(\mathbf{u})\right)-\operatorname{Prob}\left(\mathbf{Y} \in \mathcal{B}_{\Delta}(\mathbf{u})\right)\right| \\
& =\mid \sum_{k=0}^{2 M} \sum_{i_{1}=1}^{2 M} \sum_{i_{2}=i_{1}+1}^{2 M} \ldots \sum_{i_{k}=i_{k-1}+1}^{2 M}(-1)^{k}\left\{T^{(N)}\left(k, i_{1}, i_{2}, \cdots, i_{k}, \mathbf{u}, \Delta\right)-\operatorname{Prob}\left(Y_{l} \leq \sqrt{E_{l}} u_{l}-\Delta \forall l \in\left\{i_{1}, i_{2}, \cdots, i_{k}\right\},\right.\right. \\
& \left.\left.\quad Y_{l} \leq \sqrt{E_{l}} u_{l}+\Delta \forall l \notin\left\{i_{1}, i_{2}, \cdots, i_{k}\right\}\right)\right\} \mid \\
& \leq \sum_{k=0}^{2 M} \sum_{i_{1}=1}^{2 M} \sum_{i_{2}=i_{1}+1}^{2 M} \ldots \sum_{i_{k}=i_{k-1}+1}^{2 M} \mid\left\{T^{(N)}\left(k, i_{1}, i_{2}, \cdots, i_{k}, \mathbf{u}, \Delta\right)-\operatorname{Prob}\left(Y_{l} \leq \sqrt{E_{l}} u_{l}-\Delta \forall l \in\left\{i_{1}, i_{2}, \cdots, i_{k}\right\},\right.\right. \\
& \leq \sum_{k=0}^{2 M} \sum_{i_{1}=1}^{2 M} \sum_{i_{2}=i_{1}+1}^{2 M} \cdots \sum_{i_{k}=i_{k-1}+1}^{2 M} \delta=2^{2 M} \delta .
\end{aligned}
$$

$T^{(N)}\left(k, i_{1}, i_{2}, \cdots, i_{k}, \mathbf{u}, \Delta\right)$, there exists a corresponding positive integer $N\left(k, i_{1}, i_{2}, \cdots, i_{k}, \delta, \mathbf{u}, \Delta\right)$ such that (17) is satisfied for all $N \geq N\left(k, i_{1}, i_{2}, \cdots, i_{k}, \delta, \mathbf{u}, \Delta\right)$. We then choose a positive integer $g\left(\left\{\mathbf{H}_{N}\right\}, \mathbf{u}, \Delta, \delta\right)$ given by (18). Combining (15), (16) and (17), for all $N \geq g\left(\left\{\mathbf{H}_{N}\right\}, \mathbf{u}, \Delta, \delta\right)$ we have (19). Since the range space (support) of $\mathbf{Y}$ is the entire space $\mathbb{R}^{2 M}$, it follows that $\operatorname{Prob}\left(\mathbf{Y} \in \mathcal{B}_{\Delta}(\mathbf{u})\right)>0$ (i.e., strictly positive) for any $\Delta>0$ and all $\mathbf{u} \in \mathcal{U}$. For the given information symbol vector $\mathbf{u}$ and $\Delta>0$, we choose a corresponding $\delta$ given by

$$
\delta(\mathbf{u}, \Delta) \triangleq \frac{1}{2} \frac{\operatorname{Prob}\left(\mathbf{Y} \in \mathcal{B}_{\Delta}(\mathbf{u})\right)}{2^{2 M}}>0
$$

From (19) and (20) it now follows that, for all $N>$ $g\left(\left\{\mathbf{H}_{N}\right\}, \mathbf{u}, \Delta, \delta(\mathbf{u}, \Delta)\right)$ we have

$$
\begin{aligned}
\left|\operatorname{Prob}\left(\mathbf{z}_{N} \in \mathcal{B}_{\Delta}(\mathbf{u})\right)-\operatorname{Prob}\left(\mathbf{Y} \in \mathcal{B}_{\Delta}(\mathbf{u})\right)\right| & \leq 2^{2 M} \delta(\mathbf{u}, \Delta) \\
& =\frac{\operatorname{Prob}\left(\mathbf{Y} \in \mathcal{B}_{\Delta}(\mathbf{u})\right)}{2}
\end{aligned}
$$

which then implies that

$$
\operatorname{Prob}\left(\mathbf{z}_{N} \in \mathcal{B}_{\Delta}(\mathbf{u})\right) \geq \frac{\operatorname{Prob}\left(\mathbf{Y} \in \mathcal{B}_{\Delta}(\mathbf{u})\right)}{2}>0
$$

i.e., $\operatorname{Prob}\left(\mathbf{z}_{N} \in \mathcal{B}_{\Delta}(\mathbf{u})\right)$ is strictly positive for $N>$ $g\left(\left\{\mathbf{H}_{N}\right\}, \mathbf{u}, \Delta, \delta(\mathbf{u}, \Delta)\right)$. For a given channel sequence $\left\{\mathbf{H}_{N}\right\}$, a finite $\mathcal{U}$ and $\Delta>0$, we define the integer

$$
N\left(\left\{\mathbf{H}_{N}\right\}, \mathcal{U}, \Delta\right) \triangleq \max _{u \in \mathcal{U}} g\left(\left\{\mathbf{H}_{N}\right\}, \mathbf{u}, \Delta, \delta(\mathbf{u}, \Delta)\right) .
$$

Combining this definition with the result in (22) proves the theorem.

\section{B. Proposed CE Precoding Scheme}

For reliable communication to each user, the precoder at the BS must choose a $\Theta$ such that the MUI energy is as small as possible for each $k=1,2, \ldots, M$. This motivates us to consider the following non-linear least squares (NLS) problem, which for a given $\mathbf{u}$ to be communicated, finds the transmit phase angles that minimize the sum of the MUI energy for all users:

$$
\begin{array}{r}
\Theta^{u}=\left(\theta_{1}^{u}, \cdots, \theta_{N}^{u}\right)=\arg \min _{\theta_{i} \in[-\pi, \pi), i=1, \ldots, N} g(\Theta, \mathbf{u}) \\
g(\Theta, \mathbf{u}) \triangleq \sum_{k=1}^{M}\left|s_{k}\right|^{2}=\sum_{k=1}^{M}\left|\frac{\sum_{i=1}^{N} h_{k, i} e^{j \theta_{i}}}{\sqrt{N}}-\sqrt{E_{k}} u_{k}\right|^{2} .
\end{array}
$$

This NLS problem is non-convex and has multiple local minima. However, as the ratio $N / M$ becomes large, due to the large number of extra degrees of freedom $(N-M)$, the value of the objective function $g(\Theta, \mathbf{u})$ at most local minima has been observed to be small, enabling gradient descent based methods to be used. ${ }^{3}$ However, due to the slow convergence of gradient descent based methods, we propose a novel iterative method, which has been experimentally observed to achieve similar performance but with a significantly faster convergence.

\footnotetext{
${ }^{3}$ This observation is expected, since the strict positivity of the box event probability in (10) (proof of Theorem 1), implies that there are many distinct transmit phase angles $\Theta$ such that the received noise-free vector lies in a small $2 M$-dimensional cube (box) centered at the desired information symbol vector $\mathbf{u}$, i.e., the MUI energy at each user is small for many different $\Theta$.
} 
In the proposed iterative method to solve (24), we start with the $p=0$-th iteration, where we initialize all the angles to 0 . Each iteration consists of $N$ sub-iterations. Let $\Theta^{(p, q)}=\left(\theta_{1}^{(p, q)}, \cdots, \theta_{N}^{(p, q)}\right)^{T}$ denote the phase angle vector after the $q$-th sub-iteration $(q=1,2, \ldots, N)$ of the $p$-th iteration (subsequently we shall refer to the $q$-th sub-iteration of the $p$-th iteration as the $(p, q)$-th iteration). After the $(p, q)$ th iteration, the algorithm moves either to the $(p, q+1)$-th iteration (if $q<N)$, or else it moves to the $(p+1,1)$-th iteration. In general, in the $(p, q+1)$-th iteration, the algorithm attempts to reduce the current value of the objective function i.e., $g\left(\Theta^{(p, q)}, \mathbf{u}\right)$ by only modifying the $(q+1)$-th phase angle (i.e., $\left.\theta_{q+1}^{(p, q)}\right)$ while keeping the other phase angles fixed to their values from the previous iteration. The new phase angles after the $(p, q+1)$-th iteration, are therefore given by

$$
\begin{aligned}
& \theta_{q+1}^{(p, q+1)}=\arg \min \quad g(\Theta, \mathbf{u}) \\
& \Theta=\left(\theta_{1}^{(p, q)}, \cdots, \theta_{q}^{(p, q)}, \phi, \theta_{q+2}^{(p, q)}, \cdots, \theta_{N}^{(p, q)}\right)^{T}, \phi \in[-\pi, \pi) \\
& =\pi+\arg \left(\sum _ { k = 1 } ^ { M } \frac { h _ { k , q + 1 } ^ { * } } { \sqrt { N } } \left[\left(\frac{1}{\sqrt{N}} \sum_{i=1, \neq(q+1)}^{N} h_{k, i} e^{j \theta_{i}^{(p, q)}}\right)\right.\right. \\
& \left.\left.-\sqrt{E_{k}} u_{k}\right]\right) \\
& \theta_{i}^{(p, q+1)}=\theta_{i}^{(p, q)}, i=1,2, \ldots, N, i \neq q+1 .
\end{aligned}
$$

The algorithm is terminated after a pre-defined number of iterations. We denote the phase angle vector after the last iteration by $\widehat{\Theta}^{u}=\left(\widehat{\theta}_{1}^{u}, \cdots, \widehat{\theta}_{N}^{u}\right)^{T}$. Experimentally, we have observed that, for the i.i.d. Rayleigh fading channel, with a sufficiently large $N / M$ ratio, beyond the $p=L$-th iteration (where $L$ is some constant integer), the incremental reduction in the value of the objective function is minimal. Therefore, we terminate at the $L$-th iteration. Since there are totally $L N$ sub-iterations, from the phase angle update equation in (25), it follows that the complexity of the proposed iterative algorithm is $O(M N)$.

With $\widehat{\Theta}^{u}$ as the transmitted phase angle vector, the received signal and the MUI term are given by

$$
\begin{aligned}
& y_{k}=\sqrt{P_{T}} \sqrt{E_{k}} u_{k}+\sqrt{P_{T}} \widehat{s}_{k}+w_{k}, \\
& \widehat{s}_{k} \triangleq\left(\frac{\sum_{i=1}^{N} h_{k, i} e^{j \widehat{\theta}_{i}^{u}}}{\sqrt{N}}-\sqrt{E_{k}} u_{k}\right)
\end{aligned}
$$

The received signal-to-noise-and-interference-ratio (SINR) at the $k$-th user is therefore given by

$$
\gamma_{k}\left(\mathbf{H}, E, \frac{P_{T}}{\sigma^{2}}\right)=\frac{E_{k}}{\mathbb{E}_{u_{1}, \cdots, u_{M}}\left[\left|\widehat{s}_{k}\right|^{2}\right]+\frac{\sigma^{2}}{P_{T}}}
$$

where $E \triangleq\left(E_{1}, E_{2}, \cdots, E_{M}\right)^{T}$ is the vector of information symbol energies. Note that the above SINR expression is for a given channel realization $\mathbf{H}$. For each user, we would be ideally interested to have a low value of the MUI energy $\mathbb{E}\left[\left|\widehat{s}_{k}\right|^{2}\right]$, since this would imply a larger SINR.

To illustrate the result of Theorem 1, in Fig. 1, for the i.i.d. $\mathcal{C N}(0,1)$ Rayleigh fading channel, with fixed information alphabets $\mathcal{U}_{1}=\mathcal{U}_{2}=\cdots=\mathcal{U}_{M}=(16-\mathrm{QAM}$ and Gaussian $)$ and fixed information symbol energy $E_{k}=1, k=1, \ldots, M$, we plot the ergodic (averaged over channel statistics) MUI energy $\mathbb{E}_{\mathbf{H}}\left[\left|\widehat{s}_{k}\right|^{2}\right]$ with the proposed CE precoding scheme (using the discussed iterative method for solving (24)) as a

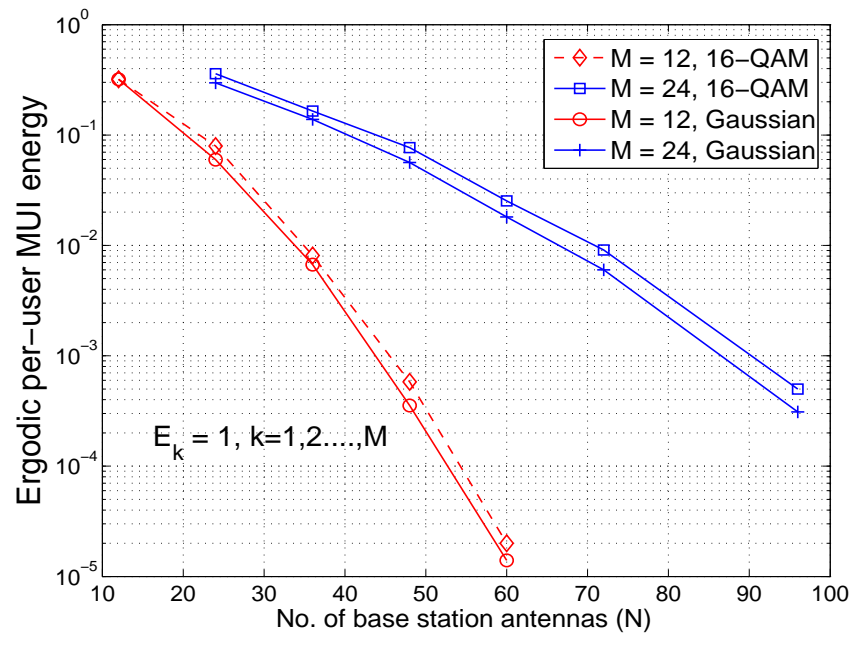

Fig. 1. Reduction in the ergodic per-user MUI energy $\mathbb{E}_{\mathbf{H}}\left[\left|\widehat{s}_{k}\right|^{2}\right]$ with increasing $N$. Fixed $M$, fixed $\mathcal{U}_{1}=\cdots=\mathcal{U}_{M}=16$-QAM, Gaussian and fixed $E_{k}=1, k=1,2, \ldots, M$. IID $\mathcal{C N}(0,1)$ Rayleigh fading.

function of increasing $N\left(\widehat{s}_{k}\right.$ is given by $\left.(26)\right) .{ }^{4}$ It is observed that, for a fixed $M$, fixed information alphabets and fixed information symbol energy, the ergodic per-user MUI energy decreases with increasing $N$. This is observed to be true, not only for a finite/discrete 16-QAM information symbol alphabet, but also for the non-discrete Gaussian information alphabet.

\section{$C$. Increasing $E_{k}$ with increasing $N$, for a fixed $M$, fixed} $\mathcal{U}_{1}, \cdots, \mathcal{U}_{M}$ and fixed desired MUI energy level

It is clear that, for a fixed $M$ and $N$, increasing $E_{k}, k=$ $1, \ldots, M$ would enlarge $\mathcal{U}$ which could then increase MUI energy level at each user (enlarging $\mathcal{U}$ might result in $\mathcal{U} \notin$ $\mathcal{M}(\mathbf{H})$ ). However, since an increase in $N$ (with fixed $M$ and $E_{k}$ ) results in a reduction of MUI (Theorem 1), it can be argued that for a fixed $M$, with increasing $N$ the information symbol energy of each user can be increased while maintaining a fixed MUI energy level at each user. Further, from (2), it is clear that for a fixed $P_{T}$ the effective SINR at the $k$ th user (i.e., $\left.E_{k} /\left(\mathbb{E}_{\mathbf{u}}\left[\left|s_{k}\right|^{2}\right]+\sigma^{2} / P_{T}\right)\right)$ will increase with increasing $N$, since $E_{k}$ can be increased while maintaining a constant MUI energy. Finally, since $\sigma^{2} / P_{T}$ increases with decreasing $P_{T}$ and the MUI energy $\left|s_{k}\right|^{2}$ is independent of $P_{T}$, by appropriately decreasing $P_{T}$ and increasing $E_{k}$ with increasing $N$ (fixed $M$ ), a constant SINR level can be maintained at each user.

This observation is based entirely on Theorem 1 (which holds for a broad class of fading channels satisfying the conditions in (6), including i.i.d. fading channels). ${ }^{5}$ The above

\footnotetext{
${ }^{4}$ We have observed that $\mathbb{E}_{\mathbf{H}}\left[\left|\widehat{s}_{k}\right|^{2}\right]$ is the same for all $k=1, \ldots, M$.

5 Since Theorem 1 holds for all finite information alphabets, the above observation is valid even for the special case when the information alphabet itself has constant amplitude symbols, e.g. PSK. However, with the proposed $\mathrm{CE}$ transmission scheme the per-antenna transmit signals have a constant envelope irrespective of the information alphabet used, and therefore using PSK type information alphabet offers no extra advantage in terms of the PAPR of the transmitted signals.
} 


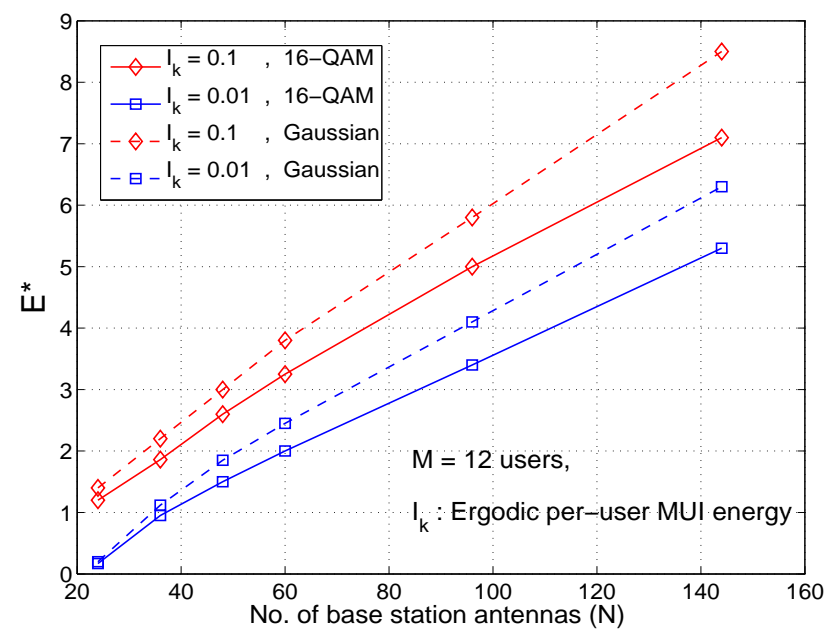

Fig. 2. $E^{\star}$ vs. $N$ for a fixed desired ergodic MUI energy level $I_{k}$ (same for each user). Fixed $M=12$, fixed $\mathcal{U}_{1}=\cdots=\mathcal{U}_{M}=16$-QAM, Gaussian. IID $\mathcal{C N}(0,1)$ Rayleigh fading.

observation implies that as long as the channel satisfies the conditions in (6), the total transmit power can be reduced without affecting user information rates, by using a sufficiently large antenna array at the BS (i.e., an achievable array gain greater than one). We illustrate this through the following example using the proposed CE precoding scheme. Let the fixed desired ergodic MUI energy level for the $k$-th user be denoted by $I_{k}, k=1,2, \cdots, M$. For the sake of simplicity we consider $\mathcal{U}_{1}=\mathcal{U}_{2}=\cdots=\mathcal{U}_{M}$. Consider

$$
E^{\star} \triangleq \max _{p>0 \mid E_{k}=p, \mathbb{E}_{\mathbf{H}}\left[\mathbb{E}_{u_{1}, \cdots, u_{M}}\left[\left|\widehat{s}_{k}\right|^{2}\right]\right]=I_{k}, k=1, \cdots, M}^{p} p
$$

which finds the highest possible equal energy of the information symbols under the constraint that the ergodic MUI energy level is fixed at $I_{k}, k=1,2, \cdots, M$. In (28), $\widehat{s}_{k}$ is given by (26). In Fig. 2, for the i.i.d. Rayleigh fading channel, for a fixed $M=12$ and a fixed $\mathcal{U}_{1}=\cdots=$ $\mathcal{U}_{M}=\left(16-\mathrm{Q} A M\right.$ and Gaussian), we plot $E^{\star}$ as a function of increasing $N$, for two different fixed desired MUI energy levels, $I_{k}=0.1$ and $I_{k}=0.01$ (same $I_{k}$ for each user ${ }^{6}$ ). From Fig. 2, it can be observed that for a fixed $M$ and fixed $\mathcal{U}_{1}, \cdots, \mathcal{U}_{M}, E^{\star}$ increases linearly with increasing $N$, while still maintaining a fixed MUI energy level at each user. At low MUI energy levels, from (27) it follows that $\gamma_{k} \approx P_{T} E_{k} / \sigma^{2}$. Since $E_{k}(k=1,2, \cdots, M)$ can be increased linearly with $N$ (while still maintaining a low MUI level), it can be argued that a desired fixed SINR level can be maintained at each user by simply reducing $P_{T}$ linearly with increasing $N$. This suggests the achievability of an $O(N)$ array power gain for the i.i.d. Rayleigh fading channel. In the next section we derive an achievable sum-rate for the proposed CE precoding scheme, using which (in Section V), for an i.i.d. Rayleigh fading channel, through simulations we show that indeed an $O(N)$ array power gain can be achieved.

\footnotetext{
${ }^{6}$ Due to same channel gain distribution and information alphabet for each user, it is observed that the ergodic MUI energy level at each user is also same if the users have equal information symbol energy.
}

\section{ACHIEVABLE InFORMATION SUM RATE}

In this section we study the ergodic information sum-rate achieved by the CE precoding scheme proposed in Section III-B. For a given channel realization $\mathbf{H}$, Gaussian information alphabets ${ }^{7,8} \mathcal{U}_{1}, \cdots, \mathcal{U}_{M}$, information symbol energies $E_{1}, \cdots, E_{M}$ and total transmit power to receiver noise ratio $P_{T} / \sigma^{2}$, the mutual information between $y_{k}$ and $u_{k}$ is given by

$$
\begin{aligned}
I\left(y_{k} ; u_{k}\right) & =h\left(u_{k}\right)-h\left(u_{k} \mid y_{k}\right) \\
& =h\left(u_{k}\right)-h\left(u_{k}-\frac{y_{k}}{\sqrt{P_{T}} \sqrt{E_{k}}} \mid y_{k}\right) \\
& \geq h\left(u_{k}\right)-h\left(u_{k}-\frac{y_{k}}{\sqrt{P_{T}} \sqrt{E_{k}}}\right)
\end{aligned}
$$

where $h(z)$ denotes the differential entropy of a continuous valued r.v. $z$. The inequality in (29) follows from the fact that conditioning of a r.v. reduces its entropy. Further, using (26) in (29) we have

$$
\begin{aligned}
I\left(y_{k} ;\right. & \left.u_{k}\right) \geq h\left(u_{k}\right)-h\left(\frac{\widehat{s}_{k}}{\sqrt{E_{k}}}+\frac{w_{k}}{\sqrt{P_{T}} \sqrt{E_{k}}}\right) \\
& =\log _{2}(\pi e)-h\left(\frac{\widehat{s}_{k}}{\sqrt{E_{k}}}+\frac{w_{k}}{\sqrt{P_{T}} \sqrt{E_{k}}}\right) \\
& \geq \log _{2}(\pi e)-\log _{2}\left(\pi e \operatorname{var}\left[\frac{\widehat{s}_{k}}{\sqrt{E_{k}}}+\frac{w_{k}}{\sqrt{P_{T}} \sqrt{E_{k}}}\right]\right) \\
& \geq \log _{2}(\pi e)-\log _{2}\left(\pi e \mathbb{E}\left[\left|\frac{\widehat{s}_{k}}{\sqrt{E_{k}}}+\frac{w_{k}}{\sqrt{P_{T}} \sqrt{E_{k}}}\right|^{2}\right]\right) \\
& =\log _{2}(\pi e)-\log _{2}\left(\pi e\left[\frac{\mathbb{E}\left[\left|\widehat{s}_{k}\right|^{2}\right]}{E_{k}}+\frac{\sigma^{2}}{P_{T} E_{k}}\right]\right) \\
& =\log _{2}\left(\gamma_{k}\left(\mathbf{H}, E, \frac{P_{T}}{\sigma^{2}}\right)\right) \\
& =R_{k}\left(\mathbf{H}, E, \frac{P_{T}}{\sigma^{2}}\right)
\end{aligned}
$$

where $R_{k}\left(\mathbf{H}, E, \frac{P_{T}}{\sigma^{2}}\right) \triangleq \log _{2}\left(\gamma_{k}\left(\mathbf{H}, E, \frac{P_{T}}{\sigma^{2}}\right)\right)$ is an achievable information rate for the $k$-th user, with the proposed CE precoding scheme. In (30), we have used the fact that the differential entropy of a complex Gaussian circular symmetric r.v. $z$ having variance $\sigma_{z}^{2}$ is $\log _{2}\left(\pi e \sigma_{z}^{2}\right)$. Further, for any complex scalar r.v. $z, \operatorname{var}[z] \triangleq \mathbb{E}\left[|z-\mathbb{E}[z]|^{2}\right]$. The second inequality in (30) follows from the fact that, for a complex scalar r.v., among all possible probability distributions having the same variance, the complex circular symmetric Gaussian distribution is the entropy maximizer [9]. The third inequality

\footnotetext{
${ }^{7}$ We restrict the discussion to Gaussian information alphabets, due to the difficulty in analyzing the information rate achieved with discrete alphabets. This is not a concern since, through Figs. 1 and 2, we have already observed that the two important results in Section III-A and III-C hold true for Gaussian alphabets as well.

${ }^{8}$ Gaussian information alphabets need not be optimal w.r.t. achieving the maximum sum-rate of a per-antenna CE constrained GBC. As an example, in [16], we have considered the capacity of a single-user MISO channel with perantenna CE constraints at the transmitter. Due to the scenario in [16] being much simpler compared to the multi-user scenario discussed here, in [16] we were able to show that the optimal capacity achieving complex alphabet is discrete-in-amplitude and uniform-in-phase (DAUIP) (i.e., non-Gaussian). However, since it appears that the analytical tools and techniques in [16] cannot be used to derive the optimal alphabet for the multiuser scenario, we restrict ourselves to Gaussian alphabets here.
} 


\begin{tabular}{|c|c|c|c|c|c|c|c|c|c|}
\hline & $\mathrm{N}=60$ & $\mathrm{~N}=80$ & $\mathrm{~N}=100$ & $\mathrm{~N}=120$ & $\mathrm{~N}=160$ & $\mathrm{~N}=200$ & $\mathrm{~N}=240$ & $\mathrm{~N}=320$ & $\mathrm{~N}=400$ \\
\hline GBC Sum Capacity Upper Bound $(\mathrm{M}=10)$ & -2.8 & -4.0 & -5.1 & -5.8 & -7.2 & -8.2 & -8.9 & -10.2 & -11.2 \\
\hline Proposed CE Precoder $(\mathrm{M}=10)$ & -0.8 & -2.1 & -3.3 & -4.1 & -5.5 & -6.5 & -7.2 & -8.6 & -9.6 \\
\hline Power Gap $(\mathrm{M}=10)$ & 2.0 & 1.9 & 1.8 & 1.7 & 1.7 & 1.7 & 1.7 & 1.6 & 1.6 \\
\hline GBC Sum Capacity Upper Bound $(\mathrm{M}=40)$ & 3.8 & 2.4 & 1.3 & 0.6 & -0.9 & -2.0 & -2.7 & -4.1 & -5.1 \\
\hline Proposed CE Precoder $(M=40)$ & 9.2 & 6.0 & 4.1 & 3.2 & 1.4 & -0.1 & -0.9 & -2.3 & -3.5 \\
\hline Power Gap $(\mathrm{M}=40)$ & 5.4 & 3.6 & 2.8 & 2.6 & 2.3 & 1.9 & 1.8 & 1.8 & 1.6 \\
\hline
\end{tabular}

Fig. 3. Minimum $P_{T} / \sigma^{2}(\mathrm{DB})$ required to achieve a per-user ergodic rate of $2 \mathrm{bpcu}$.

follows from the fact that, for any complex scalar r.v. $z$, $\operatorname{var}[z] \leq \mathbb{E}\left[|z|^{2}\right]$. From (30) it follows that an achievable ergodic information sum-rate for the GBC under the perantenna $\mathrm{CE}$ constraint, is given by

$$
R^{\mathrm{CE}}\left(E, \frac{P_{T}}{\sigma^{2}}\right) \triangleq \sum_{k=1}^{M} \mathbb{E}_{\mathbf{H}}\left[R_{k}\left(\mathbf{H}, E, \frac{P_{T}}{\sigma^{2}}\right)\right] .
$$

Subsequently, we consider the scenario where all users have the same unit energy Gaussian information alphabet and the same information symbol energy. ${ }^{9}$ Further optimization of $R^{\mathrm{CE}}\left(E, \frac{P_{T}}{\sigma^{2}}\right)$ over $E$ subject to $E_{1}=\cdots=E_{M}$, results in an achievable ergodic information sum-rate which is given by

$$
R^{\mathrm{CE}}\left(\frac{P_{T}}{\sigma^{2}}\right) \triangleq \max _{E \mid E_{1}=E_{2}=\cdots=E_{M}>0} R^{\mathrm{CE}}\left(E, \frac{P_{T}}{\sigma^{2}}\right)
$$

Since it is difficult to analyze the sum-rate expression in (32), we have studied it through exhaustive numerical simulations for an i.i.d. $\mathcal{C N}(0,1)$ Rayleigh fading channel. In the following section, we present some important observations based on these numerical experiments.

\section{Simulation Results on the aChiEvable ERGOdic INFORMATION SUM-RATE $R^{\mathrm{CE}}\left(\frac{P_{T}}{\sigma^{2}}\right)$}

All reported results are for the i.i.d. $\mathcal{C N}(0,1)$ Rayleigh fading channel. In Fig. 4, for a fixed $M$ we plot the minimum $P_{T} / \sigma^{2}$ required by the proposed CE precoder, to achieve an ergodic per-user information rate of $R^{\mathrm{CE}}\left(P_{T} / \sigma^{2}\right) / M=2$ bpcu as a function of increasing $N$ (Due to the same channel distribution for each user, we have observed that the ergodic information rate achieved by each user is $1 / M$ of the ergodic sum-rate). The minimum required $P_{T} / \sigma^{2}$ is also tabulated in Fig. 3. It is observed that, for a fixed $M$, at sufficiently large $N$, the required $P_{T} / \sigma^{2}$ reduces by roughly $3 \mathrm{~dB}$ for every doubling in $N$. This shows that, for a fixed $M$, an array power gain of $O(N)$ can indeed be achieved even under the stringent per-antenna CE constraint. For the sake of comparison, we have also plotted a lower bound on the $P_{T} / \sigma^{2}$ required to achieve a per-user ergodic rate of $2 \mathrm{bpcu}$ under the APC constraint (we have used the cooperative upper bound on the GBC sum-capacity [10]). ${ }^{10}$ We observe that, for large $N$ and

\footnotetext{
${ }^{9} \mathrm{We}$ impose this constraint so as to reduce the number of parameters involved, thereby simplifying the study of achievable rates in a multi-user GBC with per-antenna CE transmission. Nevertheless, for the i.i.d. Rayleigh fading channel with each user having the same Gaussian information alphabet, it is expected that the optimal $E$ which maximizes the ergodic sum-rate in (31), has equal components.

${ }^{10}$ The cooperative upper bound on the GBC sum capacity gives a lower bound on the $P_{T} / \sigma^{2}$ required by a GBC sum-capacity achieving scheme to achieve a given desired ergodic information sum-rate.
}

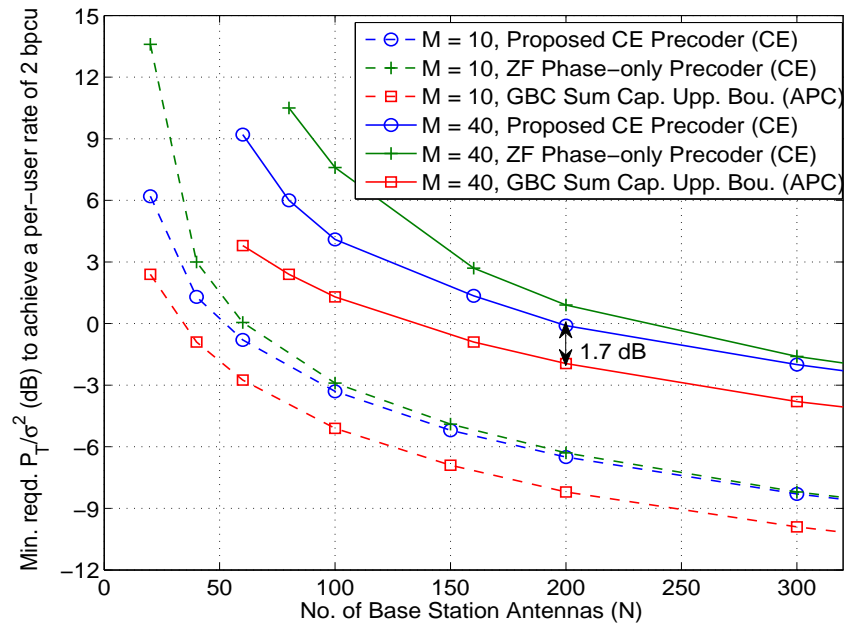

Fig. 4. Required $P_{T} / \sigma^{2}$ vs. $N$, to achieve a fixed desired ergodic peruser rate $=2$ bpcu. Gaussian information alphabets $\mathcal{U}_{1}=\cdots=\mathcal{U}_{M}$. IID $\mathcal{C N}(0,1)$ Rayleigh fading.

a fixed per-user desired ergodic information rate of $2 \mathrm{bpcu}$, compared to the APC only constrained GBC, the extra total transmit power (power gap) required under the per-antenna $\mathrm{CE}$ constraint is small $(1.7 \mathrm{~dB})$.

In Fig. 4, we also consider another CE precoding scheme, where, for a given information symbol vector $\mathbf{u}$, the precoder firstly computes the zero-forcing (ZF) vector $\mathbf{x}=\mathbf{H}^{\dagger} \mathbf{u}$, $\left(\mathbf{H}^{\dagger} \triangleq \mathbf{H}^{H}\left(\mathbf{H} \mathbf{H}^{H}\right)^{-1}\right.$ is the pseudo-inverse of $\left.\mathbf{H}\right)$. Prior to transmission, each component of $\mathbf{x}$ is normalized to have a modulus equal to $\sqrt{P_{T} / N}$, i.e., the signal transmitted from the $i$-th BS antenna is $\sqrt{P_{T} / N} x_{i} /\left|x_{i}\right|$. At each user, the received signal is scaled by a fixed constant. ${ }^{11}$ We shall henceforth refer to this precoder as the ZF phase-only precoder. In Fig. 4, we observe that the $P_{T} / \sigma^{2}$ required by the proposed CE precoder is always less than that required by the ZF phase-only precoder. In fact, for moderate values of $N / M$, the proposed CE precoder requires significantly less $P_{T} / \sigma^{2}$ as compared to the ZF phase-only precoder (e.g. with $N=100, M=40$, the required $P_{T} / \sigma^{2}$ with the proposed CE precoder is roughly 3 $\mathrm{dB}$ less than that required with the ZF phase-only precoder). At very large values of $N / M$, the ZF phase-only precoder has similar performance as the proposed CE precoder. However, in terms of complexity the ZF phase-only precoder does not necessarily have a lower complexity than the proposed CE precoder. This is because, the ZF phase-only precoder needs to compute the pseudo-inverse of the channel gain matrix

\footnotetext{
${ }^{11}$ This constant is chosen in such a way that the ergodic per-user information rate is maximized. It is therefore fixed for all channel realizations and depends only upon the statistics of the channel, $P_{T} / \sigma^{2}, N$ and $M$.
} 


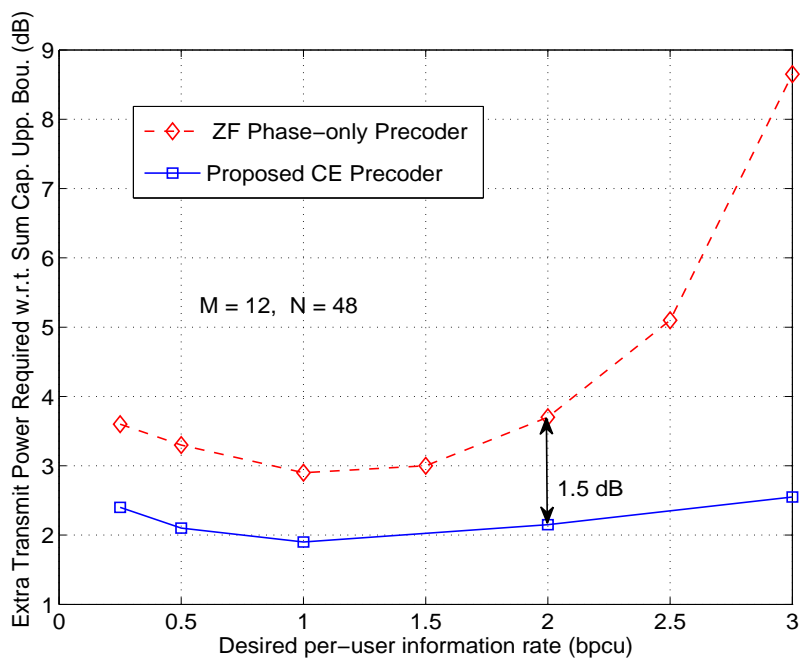

Fig. 5. The extra $P_{T} / \sigma^{2}$ (in $\mathrm{dB}$ ) required (vertical axis) by the proposed $\mathrm{CE}$ precoder and by the ZF phase-only precoder, respectively, to achieve the same ergodic per-user information rate as predicted by the GBC sumcapacity cooperative upper bound (horizontal axis). Here the number of base station antennas is $N=48$ and the number of users is $M=12$. All users use Gaussian information alphabets $\mathcal{U}_{1}=\cdots=\mathcal{U}_{M}=$ Gaussian and all channels are i.i.d. $\mathcal{C N}(0,1)$ Rayleigh fading.

(a $M \times N$ matrix) and also the matrix vector product of the pseudo-inverse times the information symbol vector $\mathbf{u}$. Computing the pseudo-inverse has a complexity of $O\left(M^{2} N\right)$ and that for the matrix vector product is $O(M N)$, resulting in a total complexity of $O\left(M^{2} N\right)$. In contrast, the proposed CE precoder does not need to compute the pseudo-inverse, and has a complexity of $O(M N)$ (see Section III-B).

To gain a better understanding of the power-efficiency of the considered CE precoders, in Fig. 5, for a fixed $N=48, M=$ 12 we plot an upper bound on the extra $P_{T} / \sigma^{2}$ required by the considered CE precoding schemes when compared to a GBC sum-capacity achieving scheme under APC, ${ }^{12}$ as a function of the desired per-user ergodic information rate (note that in Fig. 4, the desired per-user rate was fixed to $2 \mathrm{bpcu}$ ). It is observed that, for a desired ergodic per-user information rate below $2 \mathrm{bpcu}$, the ZF phase-only precoder requires roughly $1-1.5 \mathrm{~dB}$ more transmit power as compared to the proposed $\mathrm{CE}$ precoder. For rates higher than $2 \mathrm{bpcu}$, this gap increases very rapidly (at $3 \mathrm{bpcu}$, this power gap is roughly $6 \mathrm{~dB}$ ). In Fig. 6, we plot the results of a similar experiment but with $N=480, M=12$ (a very large ratio of $N / M$ ). It is observed that, the ZF phase-only precoder has similar performance as the proposed CE precoder for per-user ergodic information rates below $3 \mathrm{bpcu}$. For rates higher than $3 \mathrm{bpcu}$, the performance of the ZF phase-only precoder deteriorates rapidly, just as it did in Fig. 5. In Figs. 5 and 6, we also note that the extra total transmit power required by the proposed CE precoder (Section III-B) increases slowly w.r.t. increasing rate, and is less than $2.5 \mathrm{~dB}$ for a wide range of desired peruser information rates. From exhaustive experiments, we have concluded that, for moderate values of $N / M$, the proposed

${ }^{12}$ Since we use the cooperative upper bound to predict the $P_{T} / \sigma^{2}$ required by a GBC sum-capacity achieving scheme, the reported values of the extra $P_{T} / \sigma^{2}$ required by the considered CE precoders are infact an upper bound on the minimum extra $P_{T} / \sigma^{2}$ required.

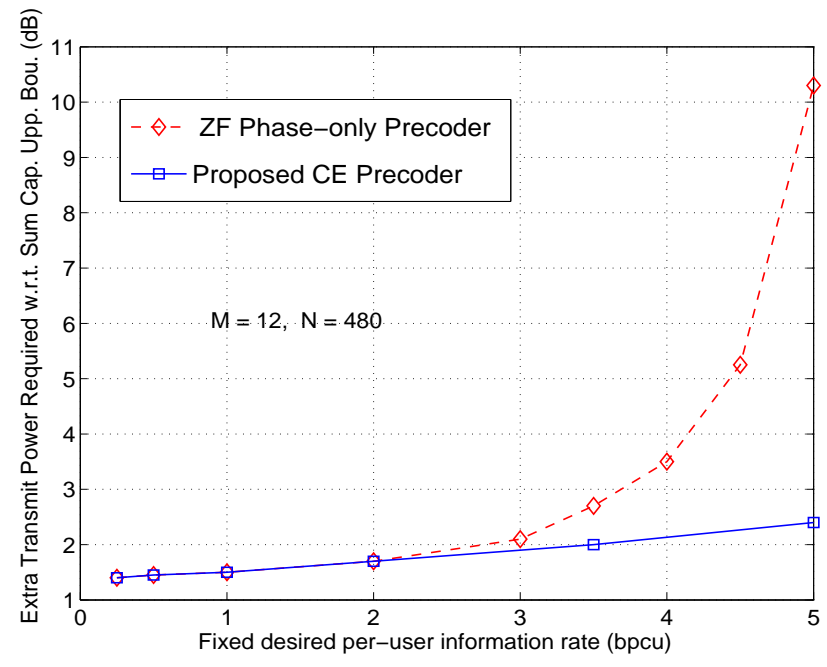

Fig. 6. Same as Fig. 5, but for $N=480$ base station antennas.

CE precoder is significantly more power efficient than the ZF phase-only precoder, whereas for very large $N / M$ both precoders have similar performance when the desired per-user ergodic information rate is below a certain threshold (beyond this threshold, the performance of the ZF phase-only precoder deteriorates).

In Fig. 4, for the proposed CE precoder, we had observed that for a fixed $M$ and fixed desired per-user information rate, with "sufficiently large" $N$, the total transmit power can be reduced linearly with increasing $N$. We next try to understand as to how "large" must $N$ be. In Fig. 7, for a fixed $M=12$ users, we plot the achievable per-user ergodic information rate under per-antenna $\mathrm{CE}$ transmission (i.e., $R^{\mathrm{CE}}\left(\frac{P_{T}}{\sigma^{2}}\right) / M$ ) as a function of increasing $N$ and $P_{T}=P_{0} / N$ (i.e., we linearly decrease $P_{T}$ with increasing $N, P_{0}=38.4$ ). It is observed that, the per-user ergodic information rate increases and approaches a limiting information rate as $N \rightarrow \infty$ (shown by the dashed curve in the figure). $P_{0}=38.4$ corresponds to a limiting per-user information rate of roughly $1.7 \mathrm{bpcu}$. This then suggests that, in the limit as $N \rightarrow \infty$, the per-user information rate remains fixed as long as $P_{T}$ is scaled down linearly with increasing $N$. A similar behaviour is observed under APC (see the GBC sum capacity upper bound curve in the figure). In Fig. 8, similar results have been illustrated for $M=24$ users and $P_{T}=P_{1} / N\left(P_{1}=72.3\right.$, corresponding to a limiting per-user information rate of roughly $1.7 \mathrm{bpcu}$ ). With regards to the question on how "large" must $N$ be, it is now clear that $N$ must at least be so large that the achievable per-user ergodic information rate is sufficiently close to its limiting information rate (i.e., in the flat region of the curve). In general, for a desired closeness to the limiting information rate, the minimum number of BS antennas required depends on $M$. Our numerical experiments suggest that, to achieve a fixed desired ratio of the per-user ergodic information rate to the limiting information rate, a channel with a large $M$ requires a large $N$ also. As an example, for a fixed ratio of 0.95 between the achievable per-user ergodic information rate and the limiting information rate, a channel with $M=12$ users requires a BS with at least $N=96$ antennas, whereas 


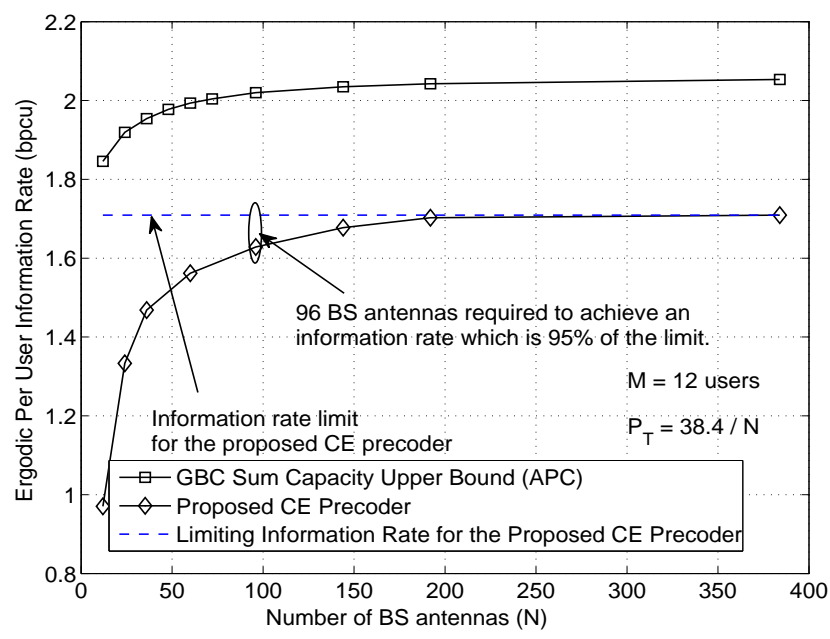

Fig. 7. Ergodic per-user information rate for a fixed $M=12$, with the total transmit power scaled down linearly with increasing $N$. Gaussian information alphabets $\mathcal{U}_{1}=\cdots=\mathcal{U}_{M}$. IID $\mathcal{C} \mathcal{N}(0,1)$ Rayleigh fading.

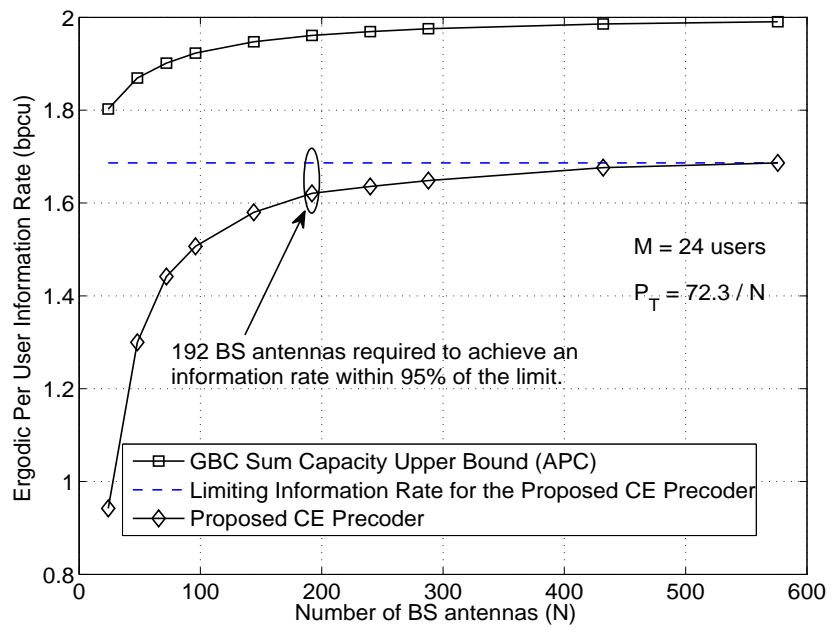

Fig. 8. Same as Fig. 7, but with a fixed $M=24$ and $P_{T}=72.3 / N$.

a channel with $M=24$ users requires a BS with at least $N=192$ antennas.

\section{CONCLUSION}

We have considered per-antenna constant envelope (CE) transmission in the downlink of multi-user MIMO systems (GBC) employing a large number of BS antennas. Under certain mild conditions on the channel, even with a stringent per-antenna $\mathrm{CE}$ constraint, array power gain can still be achieved. We have also proposed a low-complexity $\mathrm{CE}$ precoding scheme. For the proposed CE precoding scheme, through exhaustive simulations for the i.i.d. Rayleigh fading channel, we showed that, compared to an APC only constrained $\mathrm{GBC}$, the extra total transmit power required by the proposed $\mathrm{CE}$ precoder to achieve a given per-user ergodic information rate is small (less than $2 \mathrm{~dB}$ for the scenarios of interest). Typically, a non-linear power-efficient amplifier is about $4-6$ times more power-efficient than a highly linear amplifier [11]. Combining this fact with the fact that per-antenna $\mathrm{CE}$ signals require an extra $2 \mathrm{~dB}$ transmit power, we arrive at the conclusion that, for a given desired achievable information sum-rate, with sufficiently large $N$, a base station having power-efficient amplifiers with $\mathrm{CE}$ inputs would require $10 \log _{10}(4)-2.0=4.0 \mathrm{~dB}$ less total transmit power compared to a base station having highly linear powerinefficient amplifiers with high PAPR inputs.

\section{REFERENCES}

[1] F. Rusek, D. Persson, B. K. Lau, E. G. Larsson, O. Edfors, F. Tufvesson and T. L. Marzetta, "Scaling up MIMO: opportunities and challenges with very large arrays," to appear in IEEE Signal Processing Magazine.Arxiv:1201.3210v1[cs.IT].

[2] T. L. Marzetta, "Non-cooperative cellular wireless with unlimited numbers of base station antennas," IEEE. Trans. on Wireless Communications, pp. 3590-3600, vol. 9, no. 11, Nov. 2010.

[3] D. N. C. Tse, Fundamentals of Wireless Communications, Cambridge University Press, 2005

[4] Greentouch Consortium, "http://www.eweekeurope.co.uk/news/greentouchshows-low-power-wireless-19719",

[5] V. Mancuso and S. Alouf, "Reducing costs and pollution in cellular networks," IEEE Communications Mag., pp. 63-71, August 2011.

[6] W. Yu and T. Lan, "Transmitter optimization for the multi-antenna downlink with per antenna power constraints," IEEE Trans. Sig. Proc., pp. 2646-2660, vol. 55, June 2007.

[7] K. Kemai, R. Yates, G. Foschini and R. Valenzuela, "Optimum zeroforcing beamforming with per-antenna power constraints," in proc. of IEEE International Symposium on Information Theory (ISIT'07), pp. 101105, 2007.

[8] H. Q. Ngo, E. G. Larsson and T. L. Marzetta, "Energy and spectral efficiency of very large multiuser MIMO systems," submitted to IEEE Trans. on Communications. arXiv:1112.3810v2[cs.IT]

[9] T. M. Cover and J. A. Thomas, Elements of Information Theory, John Wiley and Sons, 1991.

[10] S. Vishwanath, N. Jindal, and A. Goldsmith, "Duality, achievable rates and sum-rate capacity of Gaussian MIMO broadcast channels," IEEE Transactions on Information Theory, pp. 2658-2668, vol. 49, no. 10 Oct. 2003.

[11] S. C. Cripps, RF Power Amplifiers for Wireless Communications, Artech Publishing House, 1999.

[12] V. S. Varadarajan, A useful convergence theorem, Sankhya, 20, 221-222, 1958.

[13] P. Billingsley, Probability and Measure, John Wiley and Sons, 3rd Ed., May 1995.

[14] S. Payami and F. Tufvesson, "Channel measurements and analysis for very large array systems at $2.6 \mathrm{GHz}$," in Proc. of the Sixth European Conference on Antennas and Propagation (EuCAP'12), Prague, Czech Republic, March 2012.

[15] S. K. Mohammed and E. G. Larsson, "Constant envelope precoding for power-efficient downlink wireless communication in multi-user MIMO systems using large antenna arrays," in Proc. of IEEE ICASSP'2012, Kyoto, Japan, March 25-30, 2012.

[16] S. K. Mohammed and E. G. Larsson, "Single-user beamforming in Large-Scale MISO systems with per-antenna constant-envelope constraints: The Doughnut channel", to appear in IEEE Trans. on Wireless Communications.

[17] A. K. Basu, Measure Theory and Probability, Prentice Hall of India, 1999.

\section{APPENDIX A}

\section{CONVERGENCE (IN DISTRIBUTION) OF THE SEQUENCE}

$$
\left\{\mathbf{z}_{N}\right\}
$$

The convergence in distribution of the sequence of random variables $\left\{\mathbf{z}_{N}\right\}$ (as $N \rightarrow \infty$ with fixed $M$ ) is stated and proved in Theorem 3. Its proof relies on three known results which have been stated below.

Result 1: (Multivariate Central Limit Theorem (CLT)) Let $F_{n}$ denote the joint cumulative distribution function (c.d.f.) of the $k$-dimensional real random variable $\left(X_{n}^{(1)}, \cdots, X_{n}^{(k)}\right)$, $n=1,2, \ldots$ and for each real vector $\boldsymbol{\Lambda}=\left(\lambda_{1}, \lambda_{2}, \cdots, \lambda_{k}\right)^{T}$, 
let $F_{\boldsymbol{\Lambda} n}$ be the c.d.f. of the random variable $\lambda_{1} X_{n}^{(1)}+\lambda_{2} X_{n}^{(2)}+$ $\cdots+\lambda_{k} X_{n}^{(k)}$. A necessary and sufficient condition for $F_{n}$ to converge to a limiting distribution (as $n \rightarrow \infty$ ) is that $F_{\boldsymbol{\Lambda} n}$ converges to a limit for each vector $\boldsymbol{\Lambda}$.

Proof - For details please refer to [12] .

This result basically states that, if $F$ is the joint c.d.f. of a $k$-dimensional real random variable $\left(X^{(1)}, X^{(2)}, \cdots, X^{(k)}\right)$, and if $F_{\Lambda n} \rightarrow F_{\Lambda}$ for $^{13}$ each vector $\Lambda$, then $F_{n} \rightarrow F$ as $n \rightarrow \infty$.

Result 2: (Lyapunov-CLT) Let $\left\{X_{n}\right\}, n=1,2, \ldots$ be a sequence of independent real-valued scalar random variables. Let $\mathbb{E}\left[X_{n}\right]=\mu_{n}, \mathbb{E}\left[\left(X_{n}-\mu_{n}\right)^{2}\right]=\sigma_{n}^{2}$, and for some fixed $\xi>0, \mathbb{E}\left[\left|X_{n}-\mu_{n}\right|^{2+\xi}\right]=\beta_{n}$ exists for all $n$. Furthermore let

$$
B_{n} \triangleq\left(\sum_{i=1}^{n} \beta_{i}\right)^{\frac{1}{2+\xi}}, C_{n} \triangleq\left(\sum_{i=1}^{n} \sigma_{i}^{2}\right)^{\frac{1}{2}} .
$$

Then if

$$
\lim _{n \rightarrow \infty} \frac{B_{n}}{C_{n}}=0,
$$

the c.d.f. of $Y_{n}=\frac{\sum_{i=1}^{n}\left(X_{i}-\mu_{i}\right)}{C_{n}}$ converges (in the limit as $n \rightarrow \infty$ ) to the c.d.f. of a real Gaussian random variable with mean zero and unit variance.

Proof - For details please refer to [13] .

Result 3: (Slutsky's Theorem) Let $\left\{X_{n}\right\}$ and $\left\{Y_{n}\right\}$ be a sequence of scalar random variables. If $\left\{X_{n}\right\}$ converges in distribution (as $n \rightarrow \infty$ ) to some random variable $X$, and $\left\{Y_{n}\right\}$ converges in probability to some constant $c$, then the product sequence $\left\{X_{n} Y_{n}\right\}$ converges in distribution to the random variable $c X$.

Proof - For details please refer to [17] .

Theorem 3: For any channel sequence $\left\{\mathbf{H}_{N}\right\}$ satisfying the conditions in (6), the associated sequence of random vectors $\left\{\mathbf{z}_{N}\right\}$ (defined in (8)) converges (as $N \rightarrow \infty$ with fixed $M$ ) in distribution to a multivariate $2 M$-dimensional real Gaussian random vector $X=\left(X_{1}^{I}, X_{1}^{Q}, \cdots, X_{M}^{I}, X_{M}^{Q}\right)^{T}$ with independent zero-mean components and $\operatorname{var}\left(X_{k}^{I}\right)=$ $\operatorname{var}\left(X_{k}^{Q}\right)=c_{k} / 2, k=1,2, \ldots, M$ (note that $c_{k}, k=$ $1,2, \ldots, M$ is defined in (6)).

Proof - Consider a multivariate $2 M$-dimensional real random variable $\left(X_{1}^{I}, X_{1}^{Q}, \cdots, X_{M}^{I}, X_{M}^{Q}\right)$, whose components are i.i.d. real Gaussian with mean zero and $\operatorname{var}\left(X_{k}^{I}\right)=$ $\operatorname{var}\left(X_{k}^{Q}\right)=c_{k} / 2, k=1,2, \ldots, M$. Then, for any vector $\boldsymbol{\Lambda}=\left(\lambda_{1}^{I}, \lambda_{1}^{Q}, \cdots, \lambda_{M}^{I}, \lambda_{M}^{Q}\right)^{T} \in \mathbb{R}^{2 M}$, the scalar random variable $\left(\lambda_{1}^{I} X_{1}^{I}+\lambda_{1}^{Q} X_{1}^{Q}+\cdots+\lambda_{M}^{I} X_{M}^{I}+\lambda_{M}^{Q} X_{M}^{Q}\right)$ is real Gaussian with mean zero and variance $\sum_{k=1}^{M} c_{k}\left(\left(\lambda_{k}^{I}\right)^{2}+\left(\lambda_{k}^{Q}\right)^{2}\right) / 2$.

If we can show that for any arbitrary vector $\Lambda \in \mathbb{R}^{2 M}$, the limiting distribution of $\mathbf{z}_{N}^{T} \boldsymbol{\Lambda}$ is also real Gaussian with mean zero and the same variance $\sum_{k=1}^{M} c_{k}\left(\left(\lambda_{k}^{I}\right)^{2}+\left(\lambda_{k}^{Q}\right)^{2}\right) / 2$, then using Result 1 it will follow that the c.d.f. of $\mathbf{z}_{N}$ converges to the c.d.f. of $\left(X_{1}^{I}, X_{1}^{Q}, \cdots, X_{M}^{I}, X_{M}^{Q}\right)$ as $N \rightarrow \infty$. This would then complete the proof. In the following we show that this is indeed true.

For a given $2 M$-dimensional real vector $\boldsymbol{\Lambda}=$ $\left(\lambda_{1}^{I}, \lambda_{1}^{Q}, \cdots, \lambda_{M}^{I}, \lambda_{M}^{Q}\right)^{T}$, let

$$
\zeta_{N} \triangleq \mathbf{z}_{N}^{T} \boldsymbol{\Lambda}=\sum_{k=1}^{M}\left(\lambda_{k}^{I} z_{k}^{I(N)}+\lambda_{k}^{Q} z_{k}^{Q^{(N)}}\right) .
$$

${ }^{13} F_{\boldsymbol{\Lambda}}$ is the c.d.f. of $\lambda_{1} X^{(1)}+\cdots+\lambda_{k} X^{(k)}$.
From the above definition and (8), it follows that r.v. $\zeta_{N}$ can be expressed as ${ }^{14}$

$$
\begin{aligned}
\zeta_{N} & =\sum_{i=1}^{N}\left(a_{i} \cos \left(\theta_{i}\right)+b_{i} \sin \left(\theta_{i}\right)\right) \\
& =\sum_{i=1}^{N} \sqrt{a_{i}^{2}+b_{i}^{2}} \cos \left(\theta_{i}-\tan ^{-1} \frac{b_{i}}{a_{i}}\right) \\
a_{i} & \triangleq \frac{\sum_{k=1}^{M}\left(\lambda_{k}^{I} h_{k, i}^{I^{(N)}}+\lambda_{k}^{Q} h_{k, i}^{Q^{(N)}}\right)}{\sqrt{N}}, \\
b_{i} & \triangleq \frac{\sum_{k=1}^{M}\left(\lambda_{k}^{Q} h_{k, i}^{I^{(N)}}-\lambda_{k}^{I} h_{k, i}^{Q^{(N)}}\right)}{\sqrt{N}}
\end{aligned}
$$

where $h_{k, i}^{I^{(N)}} \triangleq \operatorname{Re}\left(h_{k, i}^{(N)}\right), h_{k, i}^{Q^{(N)}} \triangleq \operatorname{Im}\left(h_{k, i}^{(N)}\right)$. We further define

$$
\eta_{i} \triangleq \sqrt{a_{i}^{2}+b_{i}^{2}} \cos \left(\theta_{i}-\tan ^{-1} \frac{b_{i}}{a_{i}}\right)
$$

Since, the phase angles $\theta_{i}, i=1,2, \ldots, N$ are independent of each other, $\eta_{i}, i=1,2, \cdots, N$ are also independent. Therefore, $\zeta_{N}$ is nothing but the sum of $N$ independent random variables. We can therefore apply the Lyapunov-CLT (Result 2) to study the convergence of the c.d.f. of $\zeta_{N}$ as $N \rightarrow \infty$.

We firstly see that $\mu_{i} \triangleq \mathbb{E}\left[\eta_{i}\right]=0$ and $\sigma_{i}^{2} \triangleq \mathbb{E}\left[\eta_{i}^{2}\right]=$ $\left(a_{i}^{2}+b_{i}^{2}\right) / 2$ since $\theta_{i}$ is uniformly distributed in $[-\pi, \pi)$. We next show that the conditions of the Lyapunov-CLT ((34) in Result 2) are satisfied with $\xi=2$. We see that

$$
\begin{aligned}
\beta_{i} & \triangleq \mathbb{E}\left[\eta_{i}^{4}\right] \\
& =\left(a_{i}^{2}+b_{i}^{2}\right)^{2} \mathbb{E}\left[\cos ^{4}\left(\theta_{i}-\tan ^{-1} \frac{b_{i}}{a_{i}}\right)\right] \\
& =\frac{3}{8}\left(a_{i}^{2}+b_{i}^{2}\right)^{2}
\end{aligned}
$$

exists for all $i$. In order that the condition in (34) is satisfied, we must show that

$$
\lim _{N \rightarrow \infty} \frac{B_{N}}{C_{N}}=0
$$

where

$$
\begin{aligned}
& B_{N} \triangleq\left(\sum_{i=1}^{N} \beta_{i}\right)^{\frac{1}{4}}=\left(\frac{3}{8} \sum_{i=1}^{N}\left(a_{i}^{2}+b_{i}^{2}\right)^{2}\right)^{\frac{1}{4}}, \\
& C_{N} \triangleq\left(\sum_{i=1}^{N} \sigma_{i}^{2}\right)^{\frac{1}{2}}=\left(\sum_{i=1}^{N}\left(a_{i}^{2}+b_{i}^{2}\right) / 2\right)^{\frac{1}{2}}
\end{aligned}
$$

As a note, from (36) it follows that both $B_{N}$ and $C_{N}$ are strictly positive for all $N \geq M$. Since $M$ is fixed, proving (39) is equivalent to proving that

$$
\lim _{N \rightarrow \infty} \frac{B_{N}^{4}}{C_{N}^{4}}=0
$$

Using (6) we firstly show that

$$
\lim _{N \rightarrow \infty} C_{N}^{2}=\frac{1}{2} \sum_{k=1}^{M} c_{k}\left(\left(\lambda_{k}^{I}\right)^{2}+\left(\lambda_{k}^{Q}\right)^{2}\right)
$$

i.e., $C_{N}^{2}$ converges to a constant as $N \rightarrow \infty$. We then show that, again under (6),

$$
\lim _{N \rightarrow \infty} B_{N}^{4}=0
$$

\footnotetext{
${ }^{14}$ Note that the randomness in $\mathbf{z}_{N}$ is only due to the random variables $\theta_{i}, i=1,2, \ldots, N$
} 


$$
\begin{aligned}
& \frac{8}{3} B_{N}^{4}=\sum_{i=1}^{N}\left(a_{i}^{2}+b_{i}^{2}\right)^{2} \\
& =\sum_{i=1}^{N}\left\{\sum_{k=1}^{M} \frac{\left|\lambda_{k}\right|^{2}\left|h_{k, i}^{(N)}\right|^{2}}{N}+2 \sum_{k=1}^{M} \sum_{l=k+1}^{M} \frac{\left(\operatorname{Re}\left(\lambda_{k}^{*} \lambda_{l}\right) \operatorname{Re}\left(h_{k, i}^{(N)^{*}} h_{l, i}^{(N)}\right)+\operatorname{Im}\left(\lambda_{k}^{*} \lambda_{l}\right) \operatorname{Im}\left(h_{k, i}^{(N)^{*}} h_{l, i}^{(N)}\right)\right)}{N}\right\}^{2} \\
& =\left\{\sum_{i=1}^{N}\left(\sum_{k=1}^{M} \frac{\left|\lambda_{k}\right|^{2}\left|h_{k, i}^{(N)}\right|^{2}}{N}\right)^{2}\right\}+4\left[\sum _ { k _ { 1 } = 1 } ^ { M } \sum _ { k _ { 2 } = 1 } ^ { M } \sum _ { l _ { 2 } = k _ { 2 } + 1 } ^ { M } \left(\left|\lambda_{k_{1}}\right|^{2} \operatorname{Re}\left(\lambda_{k_{2}}^{*} \lambda_{l_{2}}\right) \frac{\sum_{i=1}^{N}\left|h_{k_{1}, i}^{(N)}\right|^{2} \operatorname{Re}\left(h_{k_{2}, i}^{(N)} h_{l_{2}, i}^{(N)}\right)}{N^{2}}\right.\right. \\
& \left.\left.+\left|\lambda_{k_{1}}\right|^{2} \operatorname{Im}\left(\lambda_{k_{2}}^{*} \lambda_{l_{2}}\right) \frac{\sum_{i=1}^{N}\left|h_{k_{1}, i}^{(N)}\right|^{2} \operatorname{Im}\left(h_{k_{2}, i}^{(N)^{*}} h_{l_{2}, i}^{(N)}\right)}{N^{2}}\right)\right] \\
& +4 \sum_{k_{1}=1}^{M} \sum_{k_{2}=1}^{M} \sum_{l_{1}=k_{1}+1}^{M} \sum_{l_{2}=k_{2}+1}^{M}\left\{\operatorname{Re}\left(\lambda_{k_{1}}^{*} \lambda_{l_{1}}\right) \operatorname{Re}\left(\lambda_{k_{2}}^{*} \lambda_{l_{2}}\right) \frac{\sum_{i=1}^{N} \operatorname{Re}\left(h_{k_{1}, i}^{(N)^{*}} h_{l_{1}, i}^{(N)}\right) \operatorname{Re}\left(h_{k_{2}, i}^{(N)^{*}} h_{l_{2}, i}^{(N)}\right)}{N^{2}}+\right. \\
& \operatorname{Re}\left(\lambda_{k_{1}}^{*} \lambda_{l_{1}}\right) \operatorname{Im}\left(\lambda_{k_{2}}^{*} \lambda_{l_{2}}\right) \frac{\sum_{i=1}^{N} \operatorname{Re}\left(h_{k_{1}, i}^{(N)^{*}} h_{l_{1}, i}^{(N)}\right) \operatorname{Im}\left(h_{k_{2}, i}^{(N)^{*}} h_{l_{2}, i}^{(N)}\right)}{N^{2}}+ \\
& \operatorname{Im}\left(\lambda_{k_{1}}^{*} \lambda_{l_{1}}\right) \operatorname{Re}\left(\lambda_{k_{2}}^{*} \lambda_{l_{2}}\right) \frac{\sum_{i=1}^{N} \operatorname{Im}\left(h_{k_{1}, i}^{(N)^{*}} h_{l_{1}, i}^{(N)}\right) \operatorname{Re}\left(h_{k_{2}, i}^{(N)^{*}} h_{l_{2}, i}^{(N)}\right)}{N^{2}}+ \\
& \left.\operatorname{Im}\left(\lambda_{k_{1}}^{*} \lambda_{l_{1}}\right) \operatorname{Im}\left(\lambda_{k_{2}}^{*} \lambda_{l_{2}}\right) \frac{\sum_{i=1}^{N} \operatorname{Im}\left(h_{k_{1}, i}^{(N)^{*}} h_{l_{1}, i}^{(N)}\right) \operatorname{Im}\left(h_{k_{2}, i}^{(N)^{*}} h_{l_{2}, i}^{(N)}\right)}{N^{2}}\right\} \text {. }
\end{aligned}
$$

Equation (41) would then follow from (42) and (43). We next show (42). Using (40) we have $2 C_{N}^{2}=\sum_{i=1}^{N}\left(a_{i}^{2}+b_{i}^{2}\right)$. Expanding the expressions for $a_{i}$ and $b_{i}$ in $\sum_{i=1}^{\bar{N}}\left(a_{i}^{2}+b_{i}^{2}\right)$ using (36), we have

$$
\begin{aligned}
& 2 C_{N}^{2}= \sum_{k=1}^{M}\left(\left(\lambda_{k}^{I}\right)^{2}+\left(\lambda_{k}^{Q}\right)^{2}\right) \frac{\left\|\mathbf{h}_{k}^{(N)}\right\|^{2}}{N} \\
&+2 \sum_{k=1}^{M} \sum_{l=k+1}^{M}\left\{\left(\lambda_{k}^{I} \lambda_{l}^{I}+\lambda_{k}^{Q} \lambda_{l}^{Q}\right) \frac{\sum_{i=1}^{N}\left(h_{k, i}^{I^{(N)}} h_{l, i}^{I^{(N)}}+h_{k, i}^{Q^{(N)}} h_{l, i}^{Q^{(N)}}\right)}{N}\right. \\
&\left.+\left(\lambda_{k}^{I} \lambda_{l}^{Q}-\lambda_{k}^{Q} \lambda_{l}^{I}\right) \frac{\sum_{i=1}^{N}\left(h_{k, i}^{I^{(N)}} h_{l, i}^{Q^{(N)}}-h_{k, i}^{Q^{(N)}} h_{l, i}^{I^{(N)}}\right)}{N}\right\} .
\end{aligned}
$$

From As. 1 and As.3 in (6) it follows that

$$
\begin{aligned}
\lim _{N \rightarrow \infty} \frac{\sum_{i=1}^{N}\left(h_{k, i}^{I^{(N)}} h_{l, i}^{I^{(N)}}+h_{k, i}^{Q^{(N)}} h_{l, i}^{Q^{(N)}}\right)}{N} & =0, \\
\lim _{N \rightarrow \infty} \frac{\sum_{i=1}^{N}\left(h_{k, i}^{I^{(N)}} h_{l, i}^{Q^{(N)}}-h_{k, i}^{Q^{(N)}} h_{l, i}^{I^{(N)}}\right)}{N} & =0, \\
\lim _{N \rightarrow \infty} \frac{\left\|\mathbf{h}_{k}^{(N)}\right\|^{2}}{N} & =c_{k} .
\end{aligned}
$$

Using (46) in (45) and taking the limit as $N \rightarrow \infty$ we get (42) (note that $M$ is fixed). We now show (43). Before proceeding further, we define the complex numbers $\lambda_{k} \triangleq\left(\lambda_{k}^{I}+j \lambda_{k}^{Q}\right), k=$ $1,2, \ldots, M$. Expanding the expressions for $a_{i}$ and $b_{i}$ inside the summation in $B_{N}^{4}$ (see (40)) we get (44). From (As.2) in
(6) it follows that for all $k_{1}, k_{2}, l_{1}, l_{2} \in(1,2, \ldots, M)$

$$
\begin{aligned}
\lim _{N \rightarrow \infty} \frac{\sum_{i=1}^{N} \operatorname{Re}\left(h_{k_{1}, i}^{(N)^{*}} h_{l_{1}, i}^{(N)}\right) \operatorname{Re}\left(h_{k_{2}, i}^{(N)^{*}} h_{l_{2}, i}^{(N)}\right)}{N^{2}} & =0, \\
\lim _{N \rightarrow \infty} \frac{\sum_{i=1}^{N} \operatorname{Re}\left(h_{k_{1}, i}^{(N)^{*}} h_{l_{1}, i}^{(N)}\right) \operatorname{Im}\left(h_{k_{2}, i}^{(N)^{*}} h_{l_{2}, i}^{(N)}\right)}{N^{2}} & =0 \\
\lim _{N \rightarrow \infty} \frac{\sum_{i=1}^{N} \operatorname{Im}\left(h_{k_{1}, i}^{(N)^{*}} h_{l_{1}, i}^{(N)}\right) \operatorname{Re}\left(h_{k_{2}, i}^{(N)^{*}} h_{l_{2}, i}^{(N)}\right)}{N^{2}} & =0, \\
\lim _{N \rightarrow \infty} \frac{\sum_{i=1}^{N} \operatorname{Im}\left(h_{k_{1}, i}^{(N)^{*}} h_{l_{1}, i}^{(N)}\right) \operatorname{Im}\left(h_{k_{2}, i}^{(N)^{*}} h_{l_{2}, i}^{(N)}\right)}{N^{2}} & =0 \\
\lim _{N \rightarrow \infty} \frac{\sum_{i=1}^{N}\left|h_{k_{1}, i}^{(N)}\right|^{2} \operatorname{Re}\left(h_{k_{2}, i}^{(N)^{*}} h_{l_{2}, i}^{(N)}\right)}{N^{2}} & =0, \\
\lim _{N \rightarrow \infty} \frac{\sum_{i=1}^{N}\left|h_{k_{1}, i}^{(N)}\right|^{2} \operatorname{Im}\left(h_{k_{2}, i}^{(N)^{*}} h_{l_{2}, i}^{(N)}\right)}{N^{2}} & =0 .
\end{aligned}
$$

Substituting (47) into (44) and taking the limit, we have

$$
\lim _{N \rightarrow \infty} \frac{8}{3} B_{N}^{4}=\lim _{N \rightarrow \infty}\left\{\sum_{i=1}^{N}\left(\sum_{k=1}^{M} \frac{\left|\lambda_{k}\right|^{2}\left|h_{k, i}^{(N)}\right|^{2}}{N}\right)^{2}\right\}
$$

Further,

$$
\begin{aligned}
& \lim _{N \rightarrow \infty}\left\{\sum_{i=1}^{N}\left(\sum_{k=1}^{M} \frac{\left|\lambda_{k}\right|^{2}\left|h_{k, i}^{(N)}\right|^{2}}{N}\right)^{2}\right\}= \\
& \sum_{k_{1}=1}^{M} \sum_{k_{2}=1}^{M}\left(\left|\lambda_{k_{1}}\right|^{2}\left|\lambda_{k_{2}}\right|^{2} \lim _{N \rightarrow \infty}\left(\frac{\sum_{i=1}^{N}\left|h_{k_{1}, i}^{(N)}\right|^{2}\left|h_{k_{2}, i}^{(N)}\right|^{2}}{N^{2}}\right)\right) \text { (49) }
\end{aligned}
$$

From (As.2) in (6) it follows that $\lim _{N \rightarrow \infty}\left(\frac{\sum_{i=1}^{N}\left|h_{k_{1}, i}^{(N)}\right|^{2}\left|h_{k_{2}, i}^{(N)}\right|^{2}}{N^{2}}\right)=0$ and therefore using this result in (49) and (48) we get (43). From (42) it follows that $C_{N}^{4}$ converges to a positive constant as $N \rightarrow \infty$. Hence we have now shown (41), and therefore the Lyapunov-CLT conditions for the convergence of the c.d.f. of the random variable $\zeta_{N}$ are indeed satisfied.

Therefore invoking Result 2 (Lyapunov-CLT), it follows that the c.d.f. of $\zeta_{N} / C_{N}$ converges to the c.d.f. of a zero mean real 
Gaussian random variable with unit variance. Further, since $C_{N}$ converges to the constant $\sqrt{\frac{1}{2} \sum_{k=1}^{M} c_{k}\left(\left(\lambda_{k}^{I}\right)^{2}+\left(\lambda_{k}^{Q}\right)^{2}\right)}$ (see (42)), using Result 3 (Slutsky's Theorem) it follows that the c.d.f. of $\zeta_{N}$ converges to the c.d.f. of a zero mean real Gaussian random variable with variance $\frac{1}{2} \sum_{k=1}^{M} c_{k}\left(\left(\lambda_{k}^{I}\right)^{2}+\right.$ $\left.\left(\lambda_{k}^{Q}\right)^{2}\right)$.

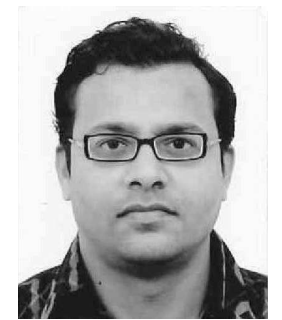

Saif Khan Mohammed (S'08-M'11) received the B.Tech degree in Computer Science and Engineering from the Indian Institute of Technology (I.I.T.), New Delhi, India, in 1998 and the Ph.D. degree from the Electrical and Communication Engineering Department, Indian Institute of Science, Bangalore, India, in 2010. Currently, he is an Assistant Professor at the Communication Systems Division (Commsys) in the Electrical Engineering Department (ISY) at Linköping University, Sweden. From 2010 to 2011, he was a Postdoctoral Researcher at Commsys. He has previously worked as a Systems and Algorithm designer in the Wireless Systems Group at Texas Instruments, Bangalore (India) (2003 - 2007). From 2000 to 2003, he worked with Ishoni Networks, Inc., Santa Clara, CA (USA), as a Senior Chip Architecture Engineer. From 1998 to 2000, he was a ASIC Design Engineer with Philips, Inc., Bangalore.

His main research interests include wireless communication using large antenna arrays, coding and signal processing for wireless communication systems, and statistical signal processing. He is a member of the IEEE, the IEEE Communication Society, the IEEE Signal Processing Society and the IEEE Information Theory Society. He is also a Technical Program Committee member for the IEEE International Conference on Communications (ICC' 2013), the IEEE Vehicular Technology Conference (VTC) in Spring 2013, and the IEEE Swedish Communication Theory Workshop (Swe-CTW) in fall 2012. Dr. Mohammed was awarded the Young Indian Researcher Fellowship by the Italian Ministry of University and Research (MIUR) for the year 200910. He has also been awarded the CENIIT research grant for the year 2012.

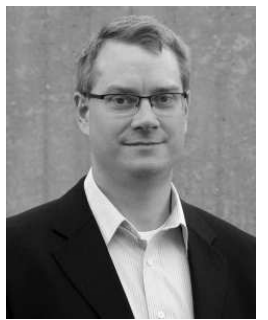

Erik Larsson received his Ph.D. degree from Uppsala University, Sweden, in 2002. Since 2007, he is Professor and Head of the Division for Communication Systems in the Department of Electrical Engineering (ISY) at Linköping University (LiU) in Linköping, Sweden. He has previously been Associate Professor (Docent) at the Royal Institute of Technology (KTH) in Stockholm, Sweden, and Assistant Professor at the University of Florida and the George Washington University, USA.

His main professional interests are within the areas of wireless communications and signal processing. He has published some 80 journal papers on these topics, he is co-author of the textbook SpaceTime Block Coding for Wireless Communications (Cambridge Univ. Press, 2003) and he holds 10 patents on wireless technology.

$\mathrm{He}$ is Associate Editor for the IEEE Transactions on Communications and he has previously been Associate Editor for several other IEEE journals. $\mathrm{He}$ is a member of the IEEE Signal Processing Society SAM and SPCOM technical committees. He is active in conference organization, most recently as the Technical Chair of the Asilomar Conference on Signals, Systems and Computers 2012 and Technical Program co-chair of the International Symposium on Turbo Codes and Iterative Information Processing 2012. 